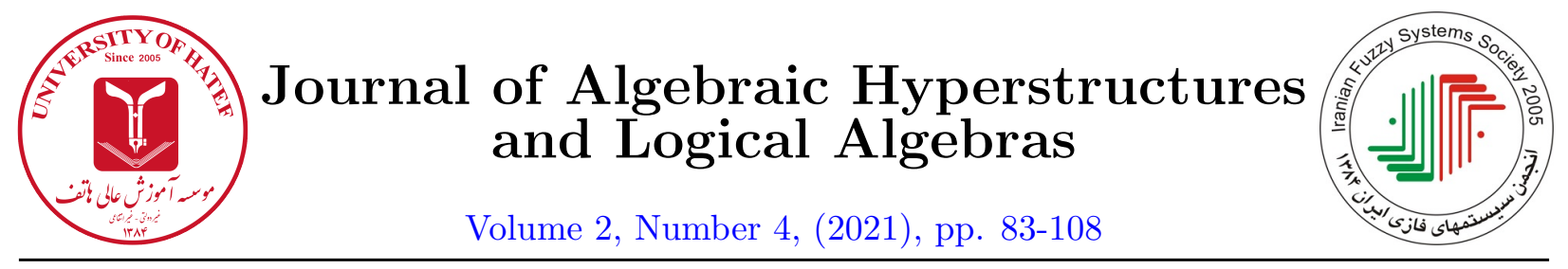

\title{
Generalized fuzzy sets with complexities and applications in decision-making problems
}

\author{
M. Khan ${ }^{1}$, A. Mukhtar ${ }^{2}$ and M. Zeeshan ${ }^{3}$ \\ ${ }^{1,2}$ Department of Mathematics, COMSATS University Islamabad, Abbottabad Campus, Pakistan \\ ${ }^{3}$ Department of Mathematics, COMSATS University Islamabad, Islamabad Campus, Pakistan \\ madadmath@yahoo.com, asmakhawaja62@gmail.com, zeeshan.msc08@gmail.com
}

\begin{abstract}
All the prevailing theories based on FS and their modifications, inconsistency, and uncertainties are involved in the form of truth grade TG whose value is also in the form of real numbers and certain user information may be lost and the decision-maker is affected by this. The principle of a complex fuzzy set (CFS) is a valuable procedure to manage inconsistent and awkward information genuine life troubles. CFS gives the TG against the value which is taken from the set of attributes in the form of a complex number whose real and unreal parts are limited to the unit interval. In this paper, we discussed some operations and formulas of set theory for complex fuzzy sets. We established the basic results of complex fuzzy sets using bounded sum, bounded product, bounded difference, simple difference, Cartesian product, algebraic product, and algebraic sums. We discussed particular examples of these operations and results. Moreover, a multicriteria decision-making (MCDM) technique is explored based on the elaborated complex fuzzy dominance matrix by using the complex fuzzy information. The application has been effectively demonstrated with numerical examples.
\end{abstract}

\section{Article Information}

Corresponding Author:

M. Zeeshan;

Received: July 2021;

Revised: September 2021;

Accepted: September 2021;

Paper type: Original.

\section{Keywords:}

Complex fuzzy sets, decisionmaking problems, complex fuzzy dominance matrix.

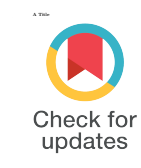

\section{Introduction}

Because of the expanding intricacy of the framework, it is hard for the leader to choose the best other option/object from a family of appealing choices. Be that as it may, it is difficult, to sum up, yet it is not staggering to accomplish the best single objective. Countless MCDM issues

https://doi.org/10.52547/HATEF.JAHLA.2.4.7 
exist in decision-making, where the rules are found to be dubious, equivocal, loose, and obscure. Therefore, the fresh set gives off an impression of being inadequate in managing this vulnerability and imprecision in the information and can be handily managed by utilizing fuzzy data. To manage such vulnerability also, vagueness, the principle of the fuzzy set (FS) was elaborated by Zadeh [21]. FS gives the truth grade (TG) against the value which is taken from the set of attributes is limited to the unit interval. After their successful utilization, certain scholars have employed it in the natural environment of separated areas. For example, interval-valued FS was explored by Zadeh [22], Torra [18] elaborated the principle of hesitant FS (HFS), and Mahmood [18] developed the theory of bipolar soft sets.

The principle of FS has been utilized in separated areas, but the principle of FS has limited applications due to its structure. Because if a person faced information in the form of TG and falsity grade (FG), then the principle of FS has been failed in certain actual life troubles. To conquer this deficiency and adjust to the uninterrupted complicacy of certain actual life troubles, a type of intuitionistic FS (IFS) was implemented at Atanassov [2]. IFS gives the information's in the form of TG and FG against the value which is taken from the set of attributes with a rule that is the sum duplet is limited to the unit interval. After their successful utilization, certain scholars have employed it in the natural environment of separated areas. For example, Atanassov [3] developed the interval-valued IFS and their application's; Garg and Rani [IV] explored similarity measures based on the transformed right-angle tringles among IFSs, Ejegwa and Onyeke [7] intuitionistic fuzzy statistical correlation algorithm, Xue et al. [1].] utilized the measure-based belief function by using the IFSs, Aydin and Enginoglu [4] proposed interval-valued intuitionistic fuzzy parametrized interval-valued intuitionistic fuzzy soft sets, Huang et al. [I2] developed the complete ranking method for interval-valued IFSs, and Ecer and Pamucer [6] initiated the MARCOS method for IFSs.

The principle of IFS has been utilized in separated areas, but the principle of IFS has limited applications due to its structure. Because if a person faced information in the form of TG and falsity grade (FG) with a condition that is the sum of duplet is exceeded from the unit interval, then the principle of IFS has been failed in certain actual life troubles. To conquer this deficiency and adjust to the uninterrupted complicacy of certain actual life troubles, a type of Pythagorean FS (PFS) was implemented by Yager [20]. PFS gives the information's in the form of TG and FG against the value which is taken from the set of attributes with a rule that is the sum of the squares of duplet is limited to the unit interval. After their successful utilization, certain scholars have employed it in the natural environment of separated areas. For example, Garg [II] explored interval-valued PFSs and their application's, Ayyildiz and Gumus [5] utilized the AHP method based on interval-valued PFSs, Ejegwa et al. [ [ $]$ implemented the correlation measures by using the PFSs, Zhao et al. [24] explored TODIM method for interval-valued PFSs, Gao et al. [24] developed the quantum Pythagorean fuzzy evidence theory, and Pan et al. [15] proposed similarity measures for PFSs.

All the prevailing theories based on FS and their modifications, inconsistency, and uncertainties are involved in the form of TG whose value is also in the form of real numbers and certain user information may be lost and the decision-maker is affected by this. To manage such vulnerability, the principle of complex FS (CFS) was elaborated by Ramot et al. [I6]. The range of CFS not only lies in the closed interval $[0,1]$, it can be broadened to the unit circle in the complex plane. In contrast to the fuzzy sets which have one-dimensional real-valued close intervals, the complex fuzzy sets have two-dimensional complex-valued disk space. The complex fuzzy set has a membership function which is designated by $Y_{x}$, for some $z^{\prime} \in U$. The membership function in $Y_{x}$ is defined as:

$$
Y_{x}\left(z^{\prime}\right)=\lambda_{x}\left(z^{\prime}\right) e^{i \psi_{x}\left(z^{\prime}\right)}, \text { where } i=\sqrt{-1},
$$


where $\lambda_{x}\left(z^{\prime}\right)$ (fuzzy set) is called the amplitude term and $\psi_{x}\left(z^{\prime}\right)$ is called the phase term. These are two real-valued functions with $\lambda_{x}\left(z^{\prime}\right) \in[0,1]$. The main characteristic of complex fuzzy sets is the existence of their phase term. If the phase term is absent then, the complex fuzzy set becomes a traditional fuzzy set. In literature, it is mentioned that to solve complicated two-dimensional problems CFS methodology is used instead of fuzzy sets. For example, periodic events like solar activity or the effect of an economic factor on one another can be more effectively visualized by utilizing a complex fuzzy set phase concept. The complex fuzzy set is also represented in the form of ordered pair as:

$$
x=\left\{\left(z^{\prime}, Y_{x}\left(z^{\prime}\right)\right) \mid z^{\prime} \in U\right\} .
$$

As Ramot et, al., [16] work on CFS and introduced the first set-theoretic operations like CF union, complement, intersection, simple difference, rotation, and reflection. Here, we built up a few fundamental operations and rules of set theory for generalized fuzzy sets with complexities. Also, we presented a few fundamental results on CFSs such as bounded sum, bounded product, bounded difference, simple difference, Cartesian product, algebraic product, and algebraic sum. We characterized specific cases with the related operation and laws.

\section{Methods}

In decision-making problems, the use of fuzzy approaches is ubiquitous. The purpose of this article is two-fold. The first half aims to present the theoretical foundations of complex fuzzy set's operations such as complex fuzzy union, complex fuzzy intersection, complex fuzzy complement, complex fuzzy bounded sum, complex fuzzy bounded product, complex fuzzy bounded difference, complex fuzzy simple difference, complex fuzzy Cartesian product, complex fuzzy algebraic product, and complex fuzzy algebraic sums and the second half aims to present these theoretical foundations and key techniques of complex fuzzy sets and complex fuzzy dominance matrix in decision-making problems. The purpose of these complex fuzzy sets and complex fuzzy dominance matrix is, to provide a new approach with useful mathematical tools to address the fundamental problem of decision-making. The generality of the fuzzy set is given special importance, illustrating how many interesting decision-making problems can be formulated as a problem of complex fuzzy sets. These applied contexts provide solid evidence of the wide applications of the complex fuzzy sets approach to model and research decision-making problems. This article will stimulate the interest in complex fuzzy sets and their application in decision-making problems.

\section{Complex fuzzy sets}

In this section, we recall the notions of complex fuzzy sets.

Definition 3.1. Let $x$ be a complex fuzzy set defined on the universal set $U$, characterized by a membership function $Y_{x}\left(z^{\prime}\right)$ and that membership function allocates any element $z^{\prime} \in U$, a complexvalued grade of membership in $x$. By definition, the range of CFS not only lies in the closed interval $[0,1]$, it can be extended to the unit circle in a complex plane. The membership function in $Y_{x}$ is defined as,

$$
Y_{x}\left(z^{\prime}\right)=\lambda_{x}\left(z^{\prime}\right) e^{i \psi_{x}\left(z^{\prime}\right)}, \text { where } i=\sqrt{-1} .
$$

where $\lambda_{x}\left(z^{\prime}\right)$ (fuzzy set) is called the amplitude term and $\psi_{x}\left(z^{\prime}\right)$ is called the phase term. These are two real-valued functions with $\lambda_{x}\left(z^{\prime}\right) \in[0,1]$. 
The complex fuzzy set $x$ is also represented in the form of ordered pair as,

$$
x=\left\{\left(z^{\prime}, Y_{x}\left(z^{\prime}\right)\right) \mid z^{\prime} \in U\right\} .
$$

\subsection{Complex fuzzy set operations}

Ramot et, al., [16] work on CFS and introduced the first set-theoretic operations like CF union, $\mathrm{CF}$ complement, and $\mathrm{CF}$ intersection, which are explained below.

Definition 3.2. [16] Suppose $\alpha_{1}^{\prime}$ is the complex fuzzy set on the universal set $U$ and the membership function of $\alpha_{1}^{\prime}$ is $Y_{\alpha_{1}^{\prime}}\left(z^{\prime}\right)=\lambda_{\alpha_{1}^{\prime}}\left(z^{\prime}\right) e^{i \psi_{\alpha_{1}^{\prime}}\left(z^{\prime}\right)}$. The complex fuzzy complement of $\alpha_{1}^{\prime}$ is denoted by $\bar{\alpha}_{1}^{\prime}$ and is specified as,

$$
Y_{\bar{\alpha}_{1}^{\prime}}\left(z^{\prime}\right)=\left[1-\lambda_{\bar{\alpha}_{1}}\left(z^{\prime}\right)\right] e^{i\left[2 \pi-\psi_{\bar{\alpha}_{1}}\left(z^{\prime}\right)\right]} .
$$

Example 3.3. Let

$$
\alpha_{1}^{\prime}=\frac{0.3 e^{i 1.2 \pi}}{-1}+\frac{0.2 e^{i \pi}}{-2}+\frac{0.1 e^{i 1.2 \pi}}{-3}
$$

The CF complement of $\alpha_{1}^{\prime}$ is

$$
\bar{\alpha}_{1}^{\prime}=\frac{0.7 e^{i 0.8 \pi}}{-1}+\frac{1.8 e^{i \pi}}{-2}+\frac{0.9 e^{i 0.8 \pi}}{-3} .
$$

Definition 3.4. [16] Suppose $\alpha_{1}^{\prime}$ and $\beta_{1}^{\prime}$ are two complex fuzzy sets. The membership functions of $\alpha_{1}^{\prime}$ and $\beta_{1}^{\prime}$ are represented by $Y_{\alpha_{1}^{\prime}}\left(z^{\prime}\right)$ and $Y_{\beta_{1}^{\prime}}\left(z^{\prime}\right)$, respectively. The union of $\alpha_{1}^{\prime}$ and $\beta_{1}^{\prime}$ is represented by $\alpha_{1}^{\prime} \cup \beta_{1}^{\prime}$ and is defined by a function

$$
Y_{\alpha_{1}^{\prime} \cup \beta_{1}^{\prime}}\left(z^{\prime}\right)=\lambda_{\alpha_{1}^{\prime} \cup \beta_{1}^{\prime}}\left(z^{\prime}\right) e^{i \psi_{\alpha_{1}^{\prime} \cup \beta_{1}^{\prime}}\left(z^{\prime}\right)}=\max \left[\lambda_{\alpha_{1}^{\prime}}\left(z^{\prime}\right), \lambda_{\beta_{1}^{\prime}}\left(z^{\prime}\right)\right] e^{i \max \left[\psi_{\alpha_{1}^{\prime}}\left(z^{\prime}\right), \psi_{\beta_{1}^{\prime}}\left(z^{\prime}\right)\right]} .
$$

Example 3.5. Let

$$
\begin{aligned}
\alpha_{1}^{\prime} & =\frac{0.1 e^{i 1.2 \pi}}{-1}+\frac{0.3 e^{i 2 \pi}}{-2}+\frac{0.9 e^{i \pi}}{-3} \\
\beta_{1}^{\prime} & =\frac{0.4 e^{i 1.4 \pi}}{-1}+\frac{0.6 e^{i 1.2 \pi}}{-2}+\frac{0.2 e^{i 1.6 \pi}}{-3} .
\end{aligned}
$$

be the two complex fuzzy sets. The union of $\alpha_{1}^{\prime}$ and $\beta_{1}^{\prime}$ is given as:

$$
\alpha_{1}^{\prime} \cup \beta_{1}^{\prime}=\frac{0.4 e^{i 1.4 \pi}}{-1}+\frac{0.6 e^{i 2 \pi}}{-2}+\frac{0.9 e^{i 1.6 \pi}}{-3} .
$$

Definition 3.6. [16] Suppose $\alpha_{1}^{\prime}$ and $\beta_{1}^{\prime}$ are two complex fuzzy sets. The membership functions of $\alpha_{1}^{\prime}$ and $\beta_{1}^{\prime}$ are represented by $Y_{\alpha_{1}^{\prime}}\left(z^{\prime}\right)$ and $Y_{\beta_{1}^{\prime}}\left(z^{\prime}\right)$, respectively. The intersection of $\alpha_{1}^{\prime}$ and $\beta_{1}^{\prime}$ is represented by $\alpha_{1}^{\prime} \cap \beta_{1}^{\prime}$ and is specified as,

$$
Y_{\alpha_{1}^{\prime} \cap \beta_{1}^{\prime}}\left(z^{\prime}\right)=\lambda_{\alpha_{1}^{\prime} \cap \beta_{1}^{\prime}}\left(z^{\prime}\right) e^{i \psi_{\alpha_{1}^{\prime} \cap \beta_{1}^{\prime}}\left(z^{\prime}\right)}=\min \left[\lambda_{\alpha_{1}^{\prime}}\left(z^{\prime}\right), \lambda_{\beta_{1}^{\prime}}\left(z^{\prime}\right)\right] e^{i \min \left[\psi_{\alpha_{1}^{\prime}}\left(z^{\prime}\right), \psi_{\beta_{1}^{\prime}}\left(z^{\prime}\right)\right]} .
$$

Example 3.7. Let

$$
\begin{aligned}
\alpha_{1}^{\prime} & =\frac{0.1 e^{i 0.1 \pi}}{-1}+\frac{0.5 e^{i 0.5 \pi}}{-2}+\frac{0.2 e^{i 1.6 \pi}}{-3} \\
\beta_{1}^{\prime} & =\frac{0.3 e^{i 0.2 \pi}}{-1}+\frac{0.9 e^{i 1.4 \pi}}{-2}+\frac{0.7 e^{i 1.8 \pi}}{-3}
\end{aligned}
$$


be two complex fuzzy sets. The CF intersection is given as:

$$
\alpha_{1}^{\prime} \cap \beta_{1}^{\prime}=\frac{0.1 e^{i 0.1 \pi}}{-1}+\frac{0.5 e^{i 0.5 \pi}}{-2}+\frac{0.2 e^{i 1.6 \pi}}{-3} .
$$

Definition 3.8. Suppose $\alpha_{1}^{\prime}$ and $\beta_{1}^{\prime}$ are two complex fuzzy sets (CFSs). $Y_{\alpha_{1}^{\prime}}\left(z^{\prime}\right)$ and $Y_{\beta_{1}^{\prime}}\left(z^{\prime}\right)$ be the membership functions of $\alpha_{1}^{\prime}$ and $\beta_{1}^{\prime}$, respectively. The simple difference of $\alpha_{1}^{\prime}$ and $\beta_{1}^{\prime}$ in CFSs is defined as,

$$
\alpha_{1}^{\prime}-\beta_{1}^{\prime}=\alpha_{1}^{\prime} \cap \bar{\beta}_{1}^{\prime}=\min \left(Y_{\alpha_{1}^{\prime}}\left(z^{\prime}\right), Y_{\bar{\beta}_{1}^{\prime}}\left(z^{\prime}\right)\right)
$$

where $Y_{\bar{\beta}_{1}^{\prime}}\left(z^{\prime}\right)$ represents the membership function of $\bar{\beta}_{1}^{\prime}$.

Example 3.9. Let

$$
\begin{aligned}
& \alpha_{1}^{\prime}=\frac{0.5 e^{i \pi}}{-1}+\frac{0.8 e^{i 1.6 \pi}}{-2}+\frac{0.7 e^{i 0.2 \pi}}{-3}, \\
& \beta_{1}^{\prime}=\frac{0.3 e^{i 1.2 \pi}}{-1}+\frac{0.2 e^{i \pi}}{-2}+\frac{0.1 e^{i 1.2 \pi}}{-3} .
\end{aligned}
$$

be two complex fuzzy sets. Then

$$
\begin{aligned}
\bar{\beta}_{1}^{\prime} & =\frac{0.7 e^{i 0.8 \pi}}{-1}+\frac{0.8 e^{i \pi}}{-2}+\frac{0.9 e^{i 0.8 \pi}}{-3} \\
\alpha_{1}^{\prime}-\beta_{1}^{\prime} & =\alpha_{1}^{\prime} \cap \bar{\beta}_{1}^{\prime}=\frac{0.5 e^{i 0.8 \pi}}{-1}+\frac{0.8 e^{i \pi}}{-2}+\frac{0.7 e^{i 0.2 \pi}}{-3} .
\end{aligned}
$$

Definition 3.10. Suppose $\alpha_{1}^{\prime}$ and $\beta_{1}^{\prime}$ are two complex fuzzy sets on the universe of discourse $U$, and the membership functions of $\alpha_{1}^{\prime}$ and $\beta_{1}^{\prime}$ are $Y_{\alpha_{1}^{\prime}}\left(z^{\prime}\right)=\lambda_{\alpha_{1}^{\prime}}\left(z^{\prime}\right) e^{i \psi_{\alpha_{1}^{\prime}}\left(z^{\prime}\right)}$ and $Y_{\beta_{1}^{\prime}}\left(z^{\prime}\right)=\lambda_{\beta_{1}^{\prime}}\left(z^{\prime}\right) e^{i \psi_{\beta_{1}^{\prime}}\left(z^{\prime}\right)}$, respectively. The bounded difference of two CFSs $\alpha_{1}^{\prime}$ and $\beta_{1}^{\prime}$ is denoted by $\alpha_{1}^{\prime} \ominus \beta_{1}^{\prime}$ and defined as,

$$
Y_{\alpha_{1}^{\prime} \ominus \beta_{1}^{\prime}}\left(z^{\prime}\right)=\lambda_{\alpha_{1}^{\prime} \ominus \beta_{1}^{\prime}}\left(z^{\prime}\right) e^{i \psi_{\alpha_{1}^{\prime} \ominus \beta_{1}^{\prime}}\left(z^{\prime}\right)}=\max \left\{0, \lambda_{\alpha_{1}^{\prime}}\left(z^{\prime}\right)-\lambda_{\beta_{1}^{\prime}}\left(z^{\prime}\right)\right\} e^{i \max \left\{0, \psi_{\alpha_{1}^{\prime}}\left(z^{\prime}\right)-\psi_{\beta_{1}^{\prime}}\left(z^{\prime}\right)\right\}} .
$$

The amplitude term in bounded difference is same as the fuzzy set. But in CFSs the major problem is to find the phase term. We have the following functions for determining the phase term, that is,

Sum:

$$
\psi_{\alpha_{1}^{\prime} \ominus \beta_{1}^{\prime}}=\psi_{\alpha_{1}^{\prime}}+\psi_{\beta_{1}^{\prime}}
$$

Max:

$$
\sigma_{\alpha_{1}^{\prime} \ominus \beta_{1}^{\prime}}=\max \left\{\psi_{\alpha_{1}^{\prime}}, \psi_{\beta_{1}^{\prime}}\right\}
$$

Min:

$$
\theta_{\alpha_{1}^{\prime} \ominus \beta_{1}^{\prime}}=\min \left\{\psi_{\alpha_{1}^{\prime}}, \psi_{\beta_{1}^{\prime}}\right\}
$$

"Winner take all"

$$
\varsigma_{\alpha_{1}^{\prime} \ominus \beta_{1}^{\prime}}=\left\{\begin{array}{cc}
\psi_{\alpha_{1}^{\prime}} ; & \lambda_{\alpha_{1}^{\prime}}\left(z^{\prime}\right)>\lambda_{\beta_{1}^{\prime}}\left(z^{\prime}\right) \\
\psi_{\beta_{1}^{\prime}} ; & \lambda_{\beta_{1}^{\prime}}\left(z^{\prime}\right)>\lambda_{\alpha_{1}^{\prime}}\left(z^{\prime}\right)
\end{array} .\right.
$$

The following functions are also applicable for determining the phase term.

\section{Difference:}

$$
\chi_{\alpha_{1}^{\prime} \ominus \beta_{1}^{\prime}}=\psi_{\alpha_{1}^{\prime}}-\psi_{\beta_{1}^{\prime}}
$$




\section{Average:}

$$
\rho_{\alpha_{1}^{\prime} \ominus \beta_{1}^{\prime}}=\frac{\psi_{\alpha_{1}^{\prime}}+\psi_{\beta_{1}^{\prime}}}{2}
$$

Weighted Average:

$$
\tau_{\alpha_{1}^{\prime} \ominus \beta_{1}^{\prime}}=\frac{\lambda_{\alpha_{1}^{\prime}} \psi_{\alpha_{1}^{\prime}}+\lambda_{\beta_{1}^{\prime}} \psi_{\beta_{1}^{\prime}}}{\lambda_{\alpha_{1}^{\prime}}+\lambda_{\beta_{1}^{\prime}}}
$$

Example 3.11. Suppose

$$
\begin{aligned}
& \alpha_{1}^{\prime}=\frac{0.5 e^{i 1.2 \pi}}{-1}+\frac{0.8 e^{i 1.8 \pi}}{-2}+\frac{0.9 e^{i 1.9 \pi}}{-3}, \\
& \beta_{1}^{\prime}=\frac{0.2 e^{i 0.3 \pi}}{-1}+\frac{0.6 e^{i 1.2 \pi}}{-2}+\frac{0.3 e^{i 0.7 \pi}}{-3},
\end{aligned}
$$

are two CFSs. Using the definition of bounded difference we get,

$$
\alpha_{1}^{\prime} \ominus \beta_{1}^{\prime}=\frac{0.3 e^{i 0.9 \pi}}{-1}+\frac{0.2 e^{i 0.6 \pi}}{-2}+\frac{0.6 e^{i 1.2 \pi}}{-3} .
$$

Definition 3.12. [23] Suppose $\alpha_{1}^{\prime}$ and $\beta_{1}^{\prime}$ are two complex fuzzy sets. The membership function of $\alpha_{1}^{\prime}$ and $\beta_{1}^{\prime}$ is represented by $Y_{\alpha_{1}^{\prime}}\left(z^{\prime}\right)$ and $Y_{\beta_{1}^{\prime}}\left(z^{\prime}\right)$, respectively. The $C F$ algebraic sum is denoted by $\alpha_{1}^{\prime}+\beta_{1}^{\prime}$ and defined as,

$Y_{\alpha_{1}^{\prime}+\beta_{1}^{\prime}}\left(z^{\prime}\right)=\lambda_{\alpha_{1}^{\prime}+\beta_{1}^{\prime}}\left(z^{\prime}\right) e^{i \psi_{\alpha_{1}^{\prime}+\beta_{1}^{\prime}}\left(z^{\prime}\right)}=\left\{\lambda_{\alpha_{1}^{\prime}}\left(z^{\prime}\right)+\lambda_{\beta_{1}^{\prime}}\left(z^{\prime}\right)-\lambda_{\alpha_{1}^{\prime}}\left(z^{\prime}\right) \cdot \lambda_{\beta_{1}^{\prime}}\left(z^{\prime}\right)\right\} e^{i 2 \pi\left\{\frac{\psi_{\alpha_{1}^{\prime}}\left(z^{\prime}\right)}{2 \pi}+\frac{\psi_{\beta_{1}^{\prime}}\left(z^{\prime}\right)}{2 \pi}-\frac{\psi_{\alpha_{1}^{\prime}}\left(z^{\prime}\right)}{2 \pi} \cdot \frac{\psi_{\beta_{1}^{\prime}}\left(z^{\prime}\right)}{2 \pi}\right\}}$.

Example 3.13. Suppose

$$
\begin{aligned}
\alpha_{1}^{\prime} & =\frac{0.3 e^{i 1.2 \pi}}{-1}+\frac{0.6 e^{i 0.8 \pi}}{-2}+\frac{0.2 e^{i 2 \pi}}{-3} \\
\beta_{1}^{\prime} & =\frac{0.4 e^{i \pi}}{-1}+\frac{0.1 e^{i 0.6 \pi}}{-2}+\frac{0.5 e^{i 2 \pi}}{-3}
\end{aligned}
$$

are two CFSs. Then the algebraic sum is given as:

$$
\alpha_{1}^{\prime} \dot{+} \beta_{1}^{\prime}=\frac{0.58 e^{i 1.6 \pi}}{-1}+\frac{0.64 e^{i 1.16 \pi}}{-2}+\frac{0.6 e^{i 2 \pi}}{-3} .
$$

Definition 3.14. [2:3] Suppose $\alpha_{1}^{\prime}$ and $\beta_{1}^{\prime}$ are two complex fuzzy sets. The membership functions of $\alpha_{1}^{\prime}$ and $\beta_{1}^{\prime}$ are represented by $Y_{\alpha_{1}^{\prime}}\left(z^{\prime}\right)$ and $Y_{\beta_{1}^{\prime}}\left(z^{\prime}\right)$, respectively. The algebraic product of $\alpha_{1}^{\prime}$ and $\beta_{1}^{\prime}$ is represented by $\alpha_{1}^{\prime} \cdot \beta_{1}^{\prime}$ and defined as,

$$
Y_{\alpha_{1}^{\prime} \cdot \beta_{1}^{\prime}}\left(z^{\prime}\right)=\lambda_{\alpha_{1}^{\prime} \cdot \beta_{1}^{\prime}}\left(z^{\prime}\right) e^{i \psi_{\alpha_{1}^{\prime} \cdot \beta_{1}^{\prime}}\left(z^{\prime}\right)}=\left\{\lambda_{\alpha_{1}^{\prime}}\left(z^{\prime}\right) \cdot \lambda_{\beta_{1}^{\prime}}\left(z^{\prime}\right)\right\} e^{i 2 \pi\left\{\frac{\psi_{\alpha_{1}^{\prime}}\left(z^{\prime}\right)}{2 \pi} \cdot \frac{\psi_{\beta_{1}^{\prime}}\left(z^{\prime}\right)}{2 \pi}\right\}} .
$$

Example 3.15. Suppose

$$
\begin{aligned}
& \alpha_{1}^{\prime}=\frac{0.5 e^{i 1.2 \pi}}{-1}+\frac{0.6 e^{i 0.3 \pi}}{-2}+\frac{0.4 e^{i 1.3 \pi}}{-3}, \\
& \beta_{1}^{\prime}=\frac{0.2 e^{i 0.1 \pi}}{-1}+\frac{0.1 e^{i \pi}}{-2}+\frac{0.8 e^{i 2 \pi}}{-3}
\end{aligned}
$$


are two complex fuzzy sets. Then the algebraic product is given as:

$$
\alpha_{1}^{\prime} \cdot \beta_{1}^{\prime}=\frac{0.1 e^{i 0.06 \pi}}{-1}+\frac{0.06 e^{i 0.15 \pi}}{-2}+\frac{0.32 e^{i 1.3 \pi}}{-3} .
$$

Definition 3.16. Let $\alpha_{1}^{\prime}$ and $\beta_{1}^{\prime}$ be two CFSs on the universe of discourse $U$ and let $Y_{\alpha_{1}^{\prime}}\left(z^{\prime}\right)$ and $Y_{\beta_{1}^{\prime}}\left(z^{\prime}\right)$ be the membership functions of $\alpha_{1}^{\prime}$ and $\beta_{1}^{\prime}$, respectively. The bounded sum of $\alpha_{1}^{\prime}$ and $\beta_{1}^{\prime}$ is represented by $\alpha_{1}^{\prime} \oplus \beta_{1}^{\prime}$ and defined as,

$$
Y_{\alpha_{1}^{\prime} \oplus \beta_{1}^{\prime}}\left(z^{\prime}\right)=\lambda_{\alpha_{1}^{\prime} \oplus \beta_{1}^{\prime}}\left(z^{\prime}\right) e^{i \psi_{\alpha_{1}^{\prime} \oplus \beta_{1}^{\prime}}\left(z^{\prime}\right)}=\min \left\{1, \lambda_{\alpha_{1}^{\prime}}\left(z^{\prime}\right)+\lambda_{\beta_{1}^{\prime}}\left(z^{\prime}\right)\right\} e^{i \min \left\{2 \pi, \psi_{\alpha_{1}^{\prime}}\left(z^{\prime}\right)+\psi_{\beta_{1}^{\prime}}\left(z^{\prime}\right)\right\}} .
$$

Example 3.17. Let

$$
\begin{aligned}
\alpha_{1}^{\prime} & =\frac{0.2 e^{i 0.9 \pi}}{-1}+\frac{0.6 e^{i 1.2 \pi}}{-2}+\frac{0.5 e^{i \pi}}{-3} \\
\beta_{1}^{\prime} & =\frac{0.3 e^{i 2 \pi}}{-1}+\frac{0.1 e^{i 0.8 \pi}}{-2}+\frac{0.9 e^{i 0.5 \pi}}{-3}
\end{aligned}
$$

be two complex fuzzy sets. Then the bounded sum is given as:

$$
\alpha_{1}^{\prime} \oplus \beta_{1}^{\prime}=\frac{0.5 e^{i 2 \pi}}{-1}+\frac{0.7 e^{i 2 \pi}}{-2}+\frac{1 e^{i 1.5 \pi}}{-3} .
$$

Definition 3.18. [2.3] Let the $N$ CFSs on the universe of discourse $U$ be $\alpha_{n}^{\prime}$, where $n=1,2, \ldots, N$ and let the membership function of $\alpha_{n}^{\prime}$ be $Y_{\alpha_{n}^{\prime}}\left(z^{\prime}\right)=\lambda_{\alpha_{n}^{\prime}}\left(z^{\prime}\right) e^{i \psi_{\alpha_{n}^{\prime}}\left(z^{\prime}\right)}$. The Cartesian product of $\alpha_{n}^{\prime}$ is defined as,

$$
\begin{aligned}
Y_{\alpha_{1}^{\prime} \times \alpha_{2}^{\prime} \times \ldots \times \alpha_{N}^{\prime}}\left(z^{\prime}\right) & =\lambda_{\alpha_{1}^{\prime} \times \alpha_{2}^{\prime} \times \ldots \times \alpha_{N}^{\prime}}\left(z^{\prime}\right) e^{i \psi_{\alpha_{1}^{\prime} \times \alpha_{2}^{\prime} \times \ldots \times \alpha_{N}^{\prime}}\left(z^{\prime}\right)} \\
& =\min \left\{\lambda_{\alpha_{1}^{\prime}}\left(z_{1}^{\prime}\right), \lambda_{\alpha_{2}^{\prime}}\left(z_{2}^{\prime}\right), \ldots, \lambda_{\alpha_{N}^{\prime}}\left(z_{N}^{\prime}\right)\right\} e^{i \min \left\{\psi_{\alpha_{1}^{\prime}}\left(z_{1}^{\prime}\right), \psi_{\alpha_{2}^{\prime}}\left(z_{2}^{\prime}\right), \ldots, \psi_{\alpha_{N}^{\prime}}\left(z_{N}^{\prime}\right)\right\}} .
\end{aligned}
$$

Example 3.19. Suppose

$$
\begin{aligned}
& \alpha_{1}^{\prime}=\frac{0.7 e^{i 0.8 \pi}}{-1}+\frac{0.1 e^{i 0.6 \pi}}{-2}+\frac{0.7 e^{i 1.2 \pi}}{-3}, \\
& \beta_{1}^{\prime}=\frac{0.4 e^{i \pi}}{-1}+\frac{0.2 e^{i 2 \pi}}{-2}+\frac{0.1 e^{i 1.3 \pi}}{-3}
\end{aligned}
$$

are two complex fuzzy sets. Using the definition of Cartesian product we have,

$$
\begin{aligned}
\alpha_{1}^{\prime} \times \beta_{1}^{\prime}= & \left\{\frac{0.4 e^{i 0.8 \pi}}{(-1,-1)}+\frac{0.2 e^{i 0.8 \pi}}{(-1,-2)}+\frac{0.1 e^{i 0.8 \pi}}{(-1,-3)}+\right. \\
& \frac{0.1 e^{i 0.6 \pi}}{(-2,-1)}+\frac{0.1 e^{i 0.6 \pi}}{(-2,-2)}+\frac{0.1 e^{i 0.6 \pi}}{(-2,-3)}+ \\
& \left.\frac{0.4 e^{i \pi}}{(-3,-1)}+\frac{0.2 e^{i 1.2 \pi}}{(-3,-2)}+\frac{0.1 e^{i 1.2 \pi}}{(-3,-3)}\right\} .
\end{aligned}
$$

Definition 3.20. Consider the two CFSs $\alpha_{1}^{\prime}$ and $\beta_{1}^{\prime}$ and membership functions of these two sets are $Y_{\alpha_{1}^{\prime}}\left(z^{\prime}\right)$ and $Y_{\beta_{1}^{\prime}}\left(z^{\prime}\right)$, respectively. Then the bounded product of $\alpha_{1}^{\prime}$ and $\beta_{1}^{\prime}$ is represented by $\alpha_{1}^{\prime} \odot \beta_{1}^{\prime}$ and is defined as,

$$
Y_{\alpha_{1}^{\prime} \odot \beta_{1}^{\prime}}\left(z^{\prime}\right)=\lambda_{\alpha_{1}^{\prime} \odot \beta_{1}^{\prime}}\left(z^{\prime}\right) e^{i \psi_{\alpha_{1}^{\prime} \odot \beta_{1}^{\prime}}\left(z^{\prime}\right)}=\max \left\{0, \lambda_{\alpha_{1}^{\prime}}\left(z^{\prime}\right)+\lambda_{\beta_{1}^{\prime}}\left(z^{\prime}\right)-1\right\} e^{i \max \left\{0, \psi_{\alpha_{1}^{\prime}}\left(z^{\prime}\right)+\psi_{\beta_{1}^{\prime}}\left(z^{\prime}\right)-2 \pi\right\}} .
$$


Example 3.21. Suppose

$$
\begin{aligned}
& \alpha_{1}^{\prime}=\frac{0.5 e^{i \pi}}{-1}+\frac{0.9 e^{i 0.8 \pi}}{-2}+\frac{0.6 e^{i 1.5 \pi}}{-3}, \\
& \beta_{1}^{\prime}=\frac{0.8 e^{i 1.6 \pi}}{-1}+\frac{0.2 e^{i 2 \pi}}{-2}+\frac{0.7 e^{i 0.8 \pi}}{-3},
\end{aligned}
$$

are two CFSs. Using the definition of bounded product we have

$$
\alpha_{1}^{\prime} \odot \beta_{1}^{\prime}=\frac{0.3 e^{i 0.6 \pi}}{-1}+\frac{0.1 e^{i 0.8 \pi}}{-2}+\frac{0.3 e^{i 0.3 \pi}}{-3} .
$$

\section{Main results}

In this section, we established the basic results of complex fuzzy sets using bounded sum, bounded product, bounded difference, simple difference, Cartesian product, algebraic product, and algebraic sums. Moreover, we discussed particular examples of these results.

Proposition 4.1. If $\alpha_{1}^{\prime}$ and $\beta_{1}^{\prime}$ are two complex fuzzy sets on the universal set $U$, then

$$
\left(\alpha_{1}^{\prime} \dot{+} \beta_{1}^{\prime}\right) \ominus \alpha_{1}^{\prime}=\bar{\alpha}_{1}^{\prime} \cdot \beta_{1}^{\prime} .
$$

Proof. Let $Y_{\alpha_{1}^{\prime}}\left(z^{\prime}\right)=\lambda_{\alpha_{1}^{\prime}}\left(z^{\prime}\right) e^{i \psi_{\alpha_{1}^{\prime}}\left(z^{\prime}\right)}$ and $Y_{\beta_{1}^{\prime}}\left(z^{\prime}\right)=\lambda_{\beta_{1}^{\prime}}\left(z^{\prime}\right) e^{i \psi_{\beta_{1}^{\prime}}\left(z^{\prime}\right)}$ be the membership functions of $\alpha_{1}^{\prime}$ and $\beta_{1}^{\prime}$. Using the definitions of algebraic sum, bounded difference, standard CF complement, and the dot product we will prove the following statement,

$$
Y_{\left(\alpha_{1}^{\prime}+\beta_{1}^{\prime}\right) \ominus \alpha_{1}^{\prime}}\left(z^{\prime}\right)=Y_{\bar{\alpha}_{1}^{\prime} \cdot \beta_{1}^{\prime}}\left(z^{\prime}\right) .
$$

The L.H.S of the above statement as:

$$
\begin{aligned}
Y_{\left(\alpha_{1}^{\prime}+\beta_{1}^{\prime}\right) \ominus \alpha_{1}^{\prime}}\left(z^{\prime}\right)= & \lambda_{\left(\alpha_{1}^{\prime}+\beta_{1}^{\prime}\right) \ominus \alpha_{1}^{\prime}}\left(z^{\prime}\right) e^{i \psi_{\left(\alpha_{1}^{\prime}+\beta_{1}^{\prime}\right) \ominus \alpha_{1}^{\prime}}\left(z^{\prime}\right)} \\
= & \max \left[0, \lambda_{\alpha_{1}^{\prime}+\beta_{1}^{\prime}}\left(z^{\prime}\right)-\lambda_{\alpha_{1}^{\prime}}\left(z^{\prime}\right)\right] e^{i \max \left[0, \psi_{\alpha_{1}^{\prime}+\beta_{1}^{\prime}}\left(z^{\prime}\right)-\psi_{\alpha_{1}^{\prime}}\left(z^{\prime}\right)\right]} \\
= & \max \left[0, \lambda_{\alpha_{1}^{\prime}}\left(z^{\prime}\right)+\lambda_{\beta_{1}^{\prime}}\left(z^{\prime}\right)-\lambda_{\alpha_{1}^{\prime}}\left(z^{\prime}\right) \cdot \lambda_{\beta_{1}^{\prime}}\left(z^{\prime}\right)-\lambda_{\alpha_{1}^{\prime}}\left(z^{\prime}\right)\right] \\
& e^{i \max \left[0,2 \pi\left[\frac{\psi_{1}^{\prime}\left(z^{\prime}\right)}{2 \pi}+\frac{\psi_{\beta_{1}^{\prime}}\left(z^{\prime}\right)}{2 \pi}-\frac{\psi_{\alpha_{1}^{\prime}}\left(z^{\prime}\right)}{2 \pi} \cdot \frac{\psi_{\beta_{1}^{\prime}}\left(z^{\prime}\right)}{2 \pi}\right]-\psi_{\alpha_{1}^{\prime}}\left(z^{\prime}\right)\right]} \\
= & \max \left[0, \lambda_{\beta_{1}^{\prime}}\left(z^{\prime}\right)-\lambda_{\alpha_{1}^{\prime}}\left(z^{\prime}\right) \cdot \lambda_{\beta_{1}^{\prime}}\left(z^{\prime}\right)\right] e^{i \max \left[0, \psi_{\beta_{1}^{\prime}}\left(z^{\prime}\right)-\frac{\psi_{\alpha_{1}^{\prime}}\left(z^{\prime}\right) \cdot \psi_{\beta_{1}^{\prime}}\left(z^{\prime}\right)}{2 \pi}\right]} \\
= & \max \left[0, \lambda_{\beta_{1}^{\prime}}\left(z^{\prime}\right)-\lambda_{\alpha_{1}^{\prime}}\left(z^{\prime}\right) \cdot \lambda_{\beta_{1}^{\prime}}\left(z^{\prime}\right)\right] e^{i \max \left[0, \frac{\left.\left[2 \pi-\psi_{\alpha_{1}^{\prime}}\left(z^{\prime}\right)\right] \psi_{\beta_{1}^{\prime}}\left(z^{\prime}\right)\right]}{2 \pi}\right]} \cdot 1
\end{aligned}
$$

Now

$$
\begin{aligned}
Y_{\bar{\alpha}_{1}^{\prime} \cdot \beta_{1}^{\prime}}\left(z^{\prime}\right) & =\lambda_{\bar{\alpha}_{1}^{\prime} \cdot \beta_{1}^{\prime}}\left(z^{\prime}\right) e^{i \psi_{\bar{\alpha}_{1}^{\prime} \cdot \beta_{1}^{\prime}}\left(z^{\prime}\right)} \\
& =\left[\lambda_{\bar{\alpha}_{1}^{\prime}}\left(z^{\prime}\right) \cdot \lambda_{\beta_{1}^{\prime}}\left(z^{\prime}\right)\right] e^{i 2 \pi\left[\frac{\psi_{\bar{\alpha}_{1}^{\prime}}\left(z^{\prime}\right)}{2 \pi} \cdot \frac{\psi_{\beta_{1}^{\prime}}\left(z^{\prime}\right)}{2 \pi}\right]} \\
& =\left[\left(1-\lambda_{\alpha_{1}^{\prime}}\left(z^{\prime}\right)\right) \lambda_{\beta_{1}^{\prime}}\left(z^{\prime}\right)\right] e^{i 2 \pi\left[\frac{2 \pi-\psi_{\alpha_{1}^{\prime}}\left(z^{\prime}\right)}{2 \pi} \cdot \frac{\psi_{\beta_{1}^{\prime}}\left(z^{\prime}\right)}{2 \pi}\right]} \\
Y_{\bar{\alpha}_{1}^{\prime} \cdot \beta_{1}^{\prime}}\left(z^{\prime}\right)= & {\left[\lambda_{\beta_{1}^{\prime}}\left(z^{\prime}\right)-\lambda_{\alpha_{1}^{\prime}}\left(z^{\prime}\right) \cdot \lambda_{\beta_{1}^{\prime}}\left(z^{\prime}\right)\right] e^{i\left[\frac{\left.\left[2 \pi-\psi_{\alpha_{1}^{\prime}}\left(z^{\prime}\right)\right] \psi_{\beta_{1}^{\prime}}\left(z^{\prime}\right)\right]}{2 \pi}\right]} . }
\end{aligned}
$$


To prove the above statement, we have the following cases:

Case I. If

$$
\lambda_{\alpha_{1}^{\prime}}\left(z^{\prime}\right) \leq \lambda_{\beta_{1}^{\prime}}\left(z^{\prime}\right) \text { and } \psi_{\alpha_{1}^{\prime}}\left(z^{\prime}\right) \leq \psi_{\beta_{1}^{\prime}}\left(z^{\prime}\right),
$$

then equation (1) can be written as

$$
Y_{\left(\alpha_{1}^{\prime}+\beta_{1}^{\prime}\right) \ominus \alpha_{1}^{\prime}}\left(z^{\prime}\right)=\left[\lambda_{\beta_{1}^{\prime}}\left(z^{\prime}\right)-\lambda_{\alpha_{1}^{\prime}}\left(z^{\prime}\right) \cdot \lambda_{\beta_{1}^{\prime}}\left(z^{\prime}\right)\right] e^{i\left[\frac{\left.\left[2 \pi-\psi_{\alpha_{1}^{\prime}}\left(z^{\prime}\right)\right] \psi_{\beta_{1}^{\prime}}\left(z^{\prime}\right)\right]}{2 \pi}\right]} .
$$

From (2) and (3), we have

$$
Y_{\left(\alpha_{1}^{\prime}+\beta_{1}^{\prime}\right) \ominus \alpha_{1}^{\prime}}\left(z^{\prime}\right)=Y_{\bar{\alpha}_{1}^{\prime} \cdot \beta_{1}^{\prime}}\left(z^{\prime}\right)
$$

Case II. If

$$
\lambda_{\alpha_{1}^{\prime}}\left(z^{\prime}\right) \geq \lambda_{\beta_{1}^{\prime}}\left(z^{\prime}\right) \text { and } \psi_{\alpha_{1}^{\prime}}\left(z^{\prime}\right) \geq \psi_{\beta_{1}^{\prime}}\left(z^{\prime}\right) .
$$

then equation (1) can be written as

$$
Y_{\left(\alpha_{1}^{\prime}+\beta_{1}^{\prime}\right) \ominus \alpha_{1}^{\prime}}\left(z^{\prime}\right)=\left[\lambda_{\beta_{1}^{\prime}}\left(z^{\prime}\right)-\lambda_{\alpha_{1}^{\prime}}\left(z^{\prime}\right) \cdot \lambda_{\beta_{1}^{\prime}}\left(z^{\prime}\right)\right] e^{i\left[\frac{\left.\left[2 \pi-\psi_{\alpha_{1}^{\prime}}\left(z^{\prime}\right)\right] \psi_{\beta_{1}^{\prime}}\left(z^{\prime}\right)\right]}{2 \pi}\right]} .
$$

From (2) and (4), we have

$$
Y_{\left(\alpha_{1}^{\prime}+\beta_{1}^{\prime}\right) \ominus \alpha_{1}^{\prime}}\left(z^{\prime}\right)=Y_{\bar{\alpha}_{1}^{\prime} \cdot \beta_{1}^{\prime}}\left(z^{\prime}\right) .
$$

Case III. If

$$
\lambda_{\alpha_{1}^{\prime}}\left(z^{\prime}\right) \leq \lambda_{\beta_{1}^{\prime}}\left(z^{\prime}\right) \text { and } \psi_{\alpha_{1}^{\prime}}\left(z^{\prime}\right) \geq \psi_{\beta_{1}^{\prime}}\left(z^{\prime}\right) .
$$

then equation (1) can be written as

$$
Y_{\left(\alpha_{1}^{\prime}+\beta_{1}^{\prime}\right) \ominus \alpha_{1}^{\prime}}\left(z^{\prime}\right)=\left[\lambda_{\beta_{1}^{\prime}}\left(z^{\prime}\right)-\lambda_{\alpha_{1}^{\prime}}\left(z^{\prime}\right) \cdot \lambda_{\beta_{1}^{\prime}}\left(z^{\prime}\right)\right] e^{i\left[\frac{\left.\left[2 \pi-\psi_{\alpha_{1}^{\prime}}\left(z^{\prime}\right)\right] \psi_{\beta_{1}^{\prime}}\left(z^{\prime}\right)\right]}{2 \pi}\right]} .
$$

From (2) and (5), we have

$$
Y_{\left(\alpha_{1}^{\prime}+\beta_{1}^{\prime}\right) \ominus \alpha_{1}^{\prime}}\left(z^{\prime}\right)=Y_{\bar{\alpha}_{1}^{\prime} \cdot \beta_{1}^{\prime}}\left(z^{\prime}\right) .
$$

Case IV. If

$$
\lambda_{\alpha_{1}^{\prime}}\left(z^{\prime}\right) \geq \lambda_{\beta_{1}^{\prime}}\left(z^{\prime}\right) \text { and } \psi_{\alpha_{1}^{\prime}}\left(z^{\prime}\right) \leq \psi_{\beta_{1}^{\prime}}\left(z^{\prime}\right),
$$

then equation (1) can be written as

$$
Y_{\left(\alpha_{1}^{\prime}+\beta_{1}^{\prime}\right) \ominus \alpha_{1}^{\prime}}\left(z^{\prime}\right)=\left[\lambda_{\beta_{1}^{\prime}}\left(z^{\prime}\right)-\lambda_{\alpha_{1}^{\prime}}\left(z^{\prime}\right) \cdot \lambda_{\beta_{1}^{\prime}}\left(z^{\prime}\right)\right] e^{i\left[\frac{\left.\left[2 \pi-\psi_{\alpha_{1}^{\prime}}\left(z^{\prime}\right)\right] \psi_{\beta_{1}^{\prime}}\left(z^{\prime}\right)\right]}{2 \pi}\right]} .
$$

From (2) and (6), we have

$$
Y_{\left(\alpha_{1}^{\prime}+\beta_{1}^{\prime}\right) \ominus \alpha_{1}^{\prime}}\left(z^{\prime}\right)=Y_{\bar{\alpha}_{1}^{\prime} \cdot \beta_{1}^{\prime}}\left(z^{\prime}\right) .
$$

Thus from the above cases, we proved that

$$
\left(\alpha_{1}^{\prime} \dot{+} \beta_{1}^{\prime}\right) \ominus \alpha_{1}^{\prime}=\bar{\alpha}_{1}^{\prime} \cdot \beta_{1}^{\prime} .
$$

Example 4.2. Suppose

$$
\begin{aligned}
& \alpha_{1}^{\prime}=\frac{0.3 e^{i 1.2 \pi}}{-1}+\frac{0.6 e^{i 0.8 \pi}}{-2}+\frac{0.2 e^{i 1.2 \pi}}{-3}, \\
& \beta_{1}^{\prime}=\frac{0.4 e^{i \pi}}{-1}+\frac{0.1 e^{i 0.6 \pi}}{-2}+\frac{0.5 e^{i 2 \pi}}{-3}
\end{aligned}
$$


are two CFSs. Then

$$
\begin{gathered}
\alpha_{1}^{\prime} \dot{+} \beta_{1}^{\prime}=\frac{0.58 e^{i 1.6 \pi}}{-1}+\frac{0.64 e^{i 1.16 \pi}}{-2}+\frac{0.6 e^{i 2 \pi}}{-3}, \\
\left(\alpha_{1}^{\prime} \dot{+} \beta_{1}^{\prime}\right) \ominus \alpha_{1}^{\prime}=\left(\frac{0.58 e^{i 1.6 \pi}}{-1}+\frac{0.64 e^{i 1.16 \pi}}{-2}+\frac{0.6 e^{i 2 \pi}}{-3}\right) \ominus\left(\frac{0.3 e^{i 1.2 \pi}}{-1}+\frac{0.6 e^{i 0.8 \pi}}{-2}+\frac{0.2 e^{i 2 \pi}}{-3}\right), \\
\left(\alpha_{1}^{\prime} \dot{+} \beta_{1}^{\prime}\right) \ominus \alpha_{1}^{\prime}=\left(\frac{0.28 e^{i 0.4 \pi}}{-1}+\frac{0.04 e^{i 0.36 \pi}}{-2}+\frac{0.4 e^{i 1.7 \pi}}{-3}\right) .
\end{gathered}
$$

Now

$$
\begin{aligned}
\bar{\alpha}_{1}^{\prime} & =\left(\frac{0.7 e^{i 0.8 \pi}}{-1}+\frac{0.4 e^{i 1.2 \pi}}{-2}+\frac{0.8 e^{i 1.7 \pi}}{-3}\right) \\
\bar{\alpha}_{1}^{\prime} \cdot \beta_{1}^{\prime} & =\left(\frac{0.28 e^{i 0.4 \pi}}{-1}+\frac{0.04 e^{i 0.36 \pi}}{-2}+\frac{0.4 e^{i 1.7 \pi}}{-3}\right) .
\end{aligned}
$$

From (1) and (2), we have

$$
\left(\alpha_{1}^{\prime} \dot{+} \beta_{1}^{\prime}\right) \ominus \alpha_{1}^{\prime}=\bar{\alpha}_{1}^{\prime} \cdot \beta_{1}^{\prime} .
$$

Proposition 4.3. If $\alpha_{1}^{\prime}, \beta_{1}^{\prime}$ and $\gamma_{1}^{\prime}$ are the three complex fuzzy sets on the universal set $U$, then

$$
\left(\alpha_{1}^{\prime} \cap \beta_{1}^{\prime}\right) \times \gamma_{1}^{\prime}=\left(\alpha_{1}^{\prime} \times \gamma_{1}^{\prime}\right) \cap\left(\beta_{1}^{\prime} \times \gamma_{1}^{\prime}\right) .
$$

Proof. Let $Y_{\alpha_{1}^{\prime}}\left(z^{\prime}\right)=\lambda_{\alpha_{1}^{\prime}}\left(z^{\prime}\right) e^{i \psi_{\alpha_{1}^{\prime}}\left(z^{\prime}\right)}, Y_{\beta_{1}^{\prime}}\left(z^{\prime}\right)=\lambda_{\beta_{1}^{\prime}}\left(z^{\prime}\right) e^{i \psi_{\beta_{1}^{\prime}}\left(z^{\prime}\right)}$ and $Y_{\gamma_{1}^{\prime}}\left(z^{\prime}\right)=\lambda_{\gamma_{1}^{\prime}}\left(z^{\prime}\right) e^{i \psi_{\gamma_{1}^{\prime}}\left(z^{\prime}\right)}$ be the membership functions of $\alpha_{1}^{\prime}, \beta_{1}^{\prime}$ and $\gamma_{1}^{\prime}$, respectively. Using the definition of complex fuzzy intersection and Cartesian product we will prove the following statement

$$
Y_{\left(\alpha_{1}^{\prime} \cap \beta_{1}^{\prime}\right) \times \gamma_{1}^{\prime}}\left(z^{\prime}\right)=Y_{\left(\alpha_{1}^{\prime} \times \gamma_{1}^{\prime}\right) \cap\left(\beta_{1}^{\prime} \times \gamma_{1}^{\prime}\right)}\left(z^{\prime}\right) .
$$

The L.H.S of the above statement is given as:

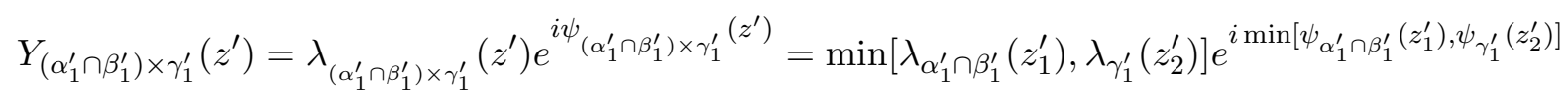

$$
\begin{aligned}
& \left.Y_{\left(\alpha_{1}^{\prime} \cap \beta_{1}^{\prime}\right) \times \gamma_{1}^{\prime}}\left(z^{\prime}\right)=\min \left[\min \left[\lambda_{\alpha_{1}^{\prime}}\left(z_{1}^{\prime}\right), \lambda_{\beta_{1}^{\prime}}\left(z_{1}^{\prime}\right)\right], \lambda_{\gamma_{1}^{\prime}}\left(z_{2}^{\prime}\right)\right]\right] e^{i \min \left[\min \left[\psi_{\alpha_{1}^{\prime}}\left(z_{1}^{\prime}\right), \psi_{\beta_{1}^{\prime}}\left(z_{1}^{\prime}\right)\right], \psi_{\gamma_{1}^{\prime}}\left(z_{2}^{\prime}\right)\right]}
\end{aligned}
$$

Now the R.H.S is given as:

$$
\begin{aligned}
Y_{\left(\alpha_{1}^{\prime} \times \gamma_{1}^{\prime}\right) \cap\left(\beta_{1}^{\prime} \times \gamma_{1}^{\prime}\right)}\left(z^{\prime}\right)= & \lambda_{\left(\alpha_{1}^{\prime} \times \gamma_{1}^{\prime}\right) \cap\left(\beta_{1}^{\prime} \times \gamma_{1}^{\prime}\right)}\left(z^{\prime}\right) e^{i \psi}\left(\alpha_{1}^{\prime} \times \gamma_{1}^{\prime}\right) \cap\left(\beta_{1}^{\prime} \times \gamma_{1}^{\prime}\right) \\
= & \min \left[\lambda_{\alpha_{1}^{\prime} \times \gamma_{1}^{\prime}}\left(z^{\prime}\right), \lambda_{\beta_{1}^{\prime} \times \gamma_{1}^{\prime}}\left(z^{\prime}\right)\right] e^{i \min \left[\psi_{\alpha_{1}^{\prime} \times \gamma_{1}^{\prime}}\left(z^{\prime}\right), \psi_{\beta_{1}^{\prime} \times \gamma_{1}^{\prime}}\left(z^{\prime}\right)\right]}, \\
Y_{\left(\alpha_{1}^{\prime} \times \gamma_{1}^{\prime}\right) \cap\left(\beta_{1}^{\prime} \times \gamma_{1}^{\prime}\right)}\left(z^{\prime}\right)= & \left.\min \left[\min \left[\lambda_{\alpha_{1}^{\prime}}\left(z_{1}^{\prime}\right), \lambda_{\gamma_{1}^{\prime}}\left(z_{2}^{\prime}\right)\right], \min \left[\lambda_{\beta_{1}^{\prime}}\left(z_{1}^{\prime}\right), \lambda_{\gamma_{1}^{\prime}}\left(z_{2}^{\prime}\right)\right)\right]\right] \\
& e^{i \min \left[\min \left[\psi_{\alpha_{1}^{\prime}}\left(z_{1}^{\prime}\right), \psi_{\gamma_{1}^{\prime}}\left(z_{2}^{\prime}\right)\right], \min \left[\psi_{\beta_{1}^{\prime}}\left(z_{1}^{\prime}\right), \psi_{\gamma_{1}^{\prime}}\left(z_{2}^{\prime}\right)\right]\right]}
\end{aligned}
$$

To prove the above statement, we have the following cases:

Case I. If

$$
\lambda_{\alpha_{1}^{\prime}}\left(z_{1}^{\prime}\right) \leq \lambda_{\beta_{1}^{\prime}}\left(z_{1}^{\prime}\right) \leq \lambda_{\gamma_{1}^{\prime}}\left(z_{2}^{\prime}\right) \text { and } \psi_{\alpha_{1}^{\prime}}\left(z_{1}^{\prime}\right) \leq \psi_{\beta_{1}^{\prime}}\left(z_{1}^{\prime}\right) \leq \psi_{\gamma_{1}^{\prime}}\left(z_{2}^{\prime}\right)
$$


then equation (1) becomes

$$
\begin{aligned}
& Y_{\left(\alpha_{1}^{\prime} \cap \beta_{1}^{\prime}\right) \times \gamma_{1}^{\prime}}\left(z^{\prime}\right)=\min \left[\lambda_{\alpha_{1}^{\prime}}\left(z_{1}^{\prime}\right), \lambda_{\gamma_{1}^{\prime}}\left(z_{2}^{\prime}\right)\right] e^{i \min \left[\psi_{\alpha_{1}^{\prime}}\left(z_{1}^{\prime}\right), \psi_{\gamma_{1}^{\prime}}\left(z_{2}^{\prime}\right)\right]}=\lambda_{\alpha_{1}^{\prime}}\left(z_{1}^{\prime}\right) e^{i \psi_{\alpha_{1}^{\prime}}\left(z_{1}^{\prime}\right)}, \\
& Y_{\left(\alpha_{1}^{\prime} \cap \beta_{1}^{\prime}\right) \times \gamma_{1}^{\prime}}\left(z^{\prime}\right)=Y_{\alpha_{1}^{\prime}}\left(z_{1}^{\prime}\right) .
\end{aligned}
$$

Also equation (2) becomes

$$
\begin{aligned}
& Y_{\left(\alpha_{1}^{\prime} \times \gamma_{1}^{\prime}\right) \cap\left(\beta_{1}^{\prime} \times \gamma_{1}^{\prime}\right)}\left(z^{\prime}\right)=\min \left[\lambda_{\alpha_{1}^{\prime}}\left(z_{1}^{\prime}\right), \lambda_{\beta_{1}^{\prime}}\left(z_{1}^{\prime}\right)\right] e^{i \min \left[\psi_{\alpha_{1}^{\prime}}\left(z_{1}^{\prime}\right), \psi_{\beta_{1}^{\prime}}\left(z_{1}^{\prime}\right)\right]}=\lambda_{\alpha_{1}^{\prime}}\left(z_{1}^{\prime}\right) e^{i \psi_{\alpha_{1}^{\prime}}\left(z_{1}^{\prime}\right)}, \\
& Y_{\left(\alpha_{1}^{\prime} \times \gamma_{1}^{\prime}\right) \cap\left(\beta_{1}^{\prime} \times \gamma_{1}^{\prime}\right)}\left(z^{\prime}\right)=Y_{\alpha_{1}^{\prime}}\left(z_{1}^{\prime}\right) .
\end{aligned}
$$

From (3) and (4), we have

$$
Y_{\left(\alpha_{1}^{\prime} \cap \beta_{1}^{\prime}\right) \times \gamma_{1}^{\prime}}\left(z^{\prime}\right)=Y_{\left(\alpha_{1}^{\prime} \times \gamma_{1}^{\prime}\right) \cap\left(\beta_{1}^{\prime} \times \gamma_{1}^{\prime}\right)}\left(z^{\prime}\right) .
$$

Case II. If

$$
\lambda_{\beta_{1}^{\prime}}\left(z_{1}^{\prime}\right) \leq \lambda_{\gamma_{1}^{\prime}}\left(z_{2}^{\prime}\right) \leq \lambda_{\alpha_{1}^{\prime}}\left(z_{1}^{\prime}\right) \text { and } \psi_{\beta_{1}^{\prime}}\left(z_{1}^{\prime}\right) \leq \psi_{\gamma_{1}^{\prime}}\left(z_{2}^{\prime}\right) \leq \psi_{\alpha_{1}^{\prime}}\left(z_{1}^{\prime}\right)
$$

then equation (1) becomes

$$
\begin{aligned}
Y_{\left(\alpha_{1}^{\prime} \cap \beta_{1}^{\prime}\right) \times \gamma_{1}^{\prime}}\left(z^{\prime}\right) & =\min \left[\lambda_{\beta_{1}^{\prime}}\left(z_{1}^{\prime}\right), \lambda_{\gamma_{1}^{\prime}}\left(z_{2}^{\prime}\right)\right] e^{i \min \left[\psi_{\beta_{1}^{\prime}}\left(z_{1}^{\prime}\right), \psi_{\gamma_{1}^{\prime}}\left(z_{2}^{\prime}\right)\right]}=\lambda_{\beta_{1}^{\prime}}\left(z_{1}^{\prime}\right) e^{i \psi_{\beta_{1}^{\prime}}\left(z_{1}^{\prime}\right)}, \\
Y_{\left(\alpha_{1}^{\prime} \cap \beta_{1}^{\prime}\right) \times \gamma_{1}^{\prime}}\left(z^{\prime}\right) & =Y_{\beta_{1}^{\prime}}\left(z_{1}^{\prime}\right) .
\end{aligned}
$$

Also, equation (2) becomes

$$
\begin{aligned}
& Y_{\left(\alpha_{1}^{\prime} \times \gamma_{1}^{\prime}\right) \cap\left(\beta_{1}^{\prime} \times \gamma_{1}^{\prime}\right)}\left(z^{\prime}\right)=\min \left[\lambda_{\gamma_{1}^{\prime}}\left(z_{2}^{\prime}\right), \lambda_{\beta_{1}^{\prime}}\left(z_{1}^{\prime}\right)\right] e^{i \min \left[\psi_{\gamma_{1}^{\prime}}\left(z_{2}^{\prime}\right), \psi_{\beta_{1}^{\prime}}\left(z_{1}^{\prime}\right)\right]}=\lambda_{\beta_{1}^{\prime}}\left(z_{1}^{\prime}\right) e^{i \psi_{\beta_{1}^{\prime}}\left(z_{1}^{\prime}\right)}, \\
& Y_{\left(\alpha_{1}^{\prime} \times \gamma_{1}^{\prime}\right) \cap\left(\beta_{1}^{\prime} \times \gamma_{1}^{\prime}\right)}\left(z^{\prime}\right)=Y_{\beta_{1}^{\prime}}\left(z_{1}^{\prime}\right) .
\end{aligned}
$$

From (5) and (6), we have

$$
Y_{\left(\alpha_{1}^{\prime} \cap \beta_{1}^{\prime}\right) \times \gamma_{1}^{\prime}}\left(z^{\prime}\right)=Y_{\left(\alpha_{1}^{\prime} \times \gamma_{1}^{\prime}\right) \cap\left(\beta_{1}^{\prime} \times \gamma_{1}^{\prime}\right)}\left(z^{\prime}\right) .
$$

Case III. If

$$
\lambda_{\gamma_{1}^{\prime}}\left(z_{2}^{\prime}\right) \leq \lambda_{\alpha_{1}^{\prime}}\left(z_{1}^{\prime}\right) \leq \lambda_{\beta_{1}^{\prime}}\left(z_{1}^{\prime}\right) \text { and } \psi_{\gamma_{1}^{\prime}}\left(z_{2}^{\prime}\right) \leq \psi_{\alpha_{1}^{\prime}}\left(z_{1}^{\prime}\right) \leq \psi_{\beta_{1}^{\prime}}\left(z_{1}^{\prime}\right)
$$

then equation (1) becomes

$$
\begin{aligned}
Y_{\left(\alpha_{1}^{\prime} \cap \beta_{1}^{\prime}\right) \times \gamma_{1}^{\prime}}\left(z^{\prime}\right) & =\min \left[\lambda_{\alpha_{1}^{\prime}}\left(z_{1}^{\prime}\right), \lambda_{\gamma_{1}^{\prime}}\left(z_{2}^{\prime}\right)\right] e^{i \min \left[\psi_{\alpha_{1}^{\prime}}\left(z_{1}^{\prime}\right), \psi_{\gamma_{1}^{\prime}}\left(z_{2}^{\prime}\right)\right]}=\lambda_{\gamma_{1}^{\prime}}\left(z_{2}^{\prime}\right) e^{i \psi_{\gamma_{1}^{\prime}}\left(z_{2}^{\prime}\right)}, \\
Y_{\left(\alpha_{1}^{\prime} \cap \beta_{1}^{\prime}\right) \times \gamma_{1}^{\prime}}\left(z^{\prime}\right) & =Y_{\gamma_{1}^{\prime}}\left(z_{2}^{\prime}\right) .
\end{aligned}
$$

Also, equation (2) becomes

$$
\begin{aligned}
& Y_{\left(\alpha_{1}^{\prime} \times \gamma_{1}^{\prime}\right) \cap\left(\beta_{1}^{\prime} \times \gamma_{1}^{\prime}\right)}\left(z^{\prime}\right)=\min \left[\lambda_{\gamma_{1}^{\prime}}\left(z_{2}^{\prime}\right), \lambda_{\gamma_{1}^{\prime}}\left(z_{2}^{\prime}\right)\right] e^{i \min \left[\psi_{\gamma_{1}^{\prime}}\left(z_{2}^{\prime}\right), \psi_{\gamma_{1}^{\prime}}\left(z_{2}^{\prime}\right)\right]}=\lambda_{\gamma_{1}^{\prime}}\left(z_{2}^{\prime}\right) e^{i \psi_{\gamma_{1}^{\prime}}\left(z_{2}^{\prime}\right)} \\
& Y_{\left(\alpha_{1}^{\prime} \times \gamma_{1}^{\prime}\right) \cap\left(\beta_{1}^{\prime} \times \gamma_{1}^{\prime}\right)}\left(z^{\prime}\right)=Y_{\gamma_{1}^{\prime}}\left(z_{2}^{\prime}\right) .
\end{aligned}
$$

From (7) and (8),we have

$$
Y_{\left(\alpha_{1}^{\prime} \cap \beta_{1}^{\prime}\right) \times \gamma_{1}^{\prime}}\left(z^{\prime}\right)=Y_{\left(\alpha_{1}^{\prime} \times \gamma_{1}^{\prime}\right) \cap\left(\beta_{1}^{\prime} \times \gamma_{1}^{\prime}\right)}\left(z^{\prime}\right)
$$




\section{Case IV. If}

$$
\lambda_{\alpha_{1}^{\prime}}\left(z_{1}^{\prime}\right) \leq \lambda_{\gamma_{1}^{\prime}}\left(z_{2}^{\prime}\right) \leq \lambda_{\beta_{1}^{\prime}}\left(z_{1}^{\prime}\right) \text { and } \psi_{\alpha_{1}^{\prime}}\left(z_{1}^{\prime}\right) \leq \psi_{\gamma_{1}^{\prime}}\left(z_{2}^{\prime}\right) \leq \psi_{\beta_{1}^{\prime}}\left(z_{1}^{\prime}\right) .
$$

then equation (1) becomes

$$
\begin{aligned}
Y_{\left(\alpha_{1}^{\prime} \cap \beta_{1}^{\prime}\right) \times \gamma_{1}^{\prime}}\left(z^{\prime}\right) & =\min \left[\lambda_{\alpha_{1}^{\prime}}\left(z_{1}^{\prime}\right), \lambda_{\gamma_{1}^{\prime}}\left(z_{2}^{\prime}\right)\right] e^{i \min \left[\psi_{\alpha_{1}^{\prime}}\left(z_{1}^{\prime}\right), \psi_{\gamma_{1}^{\prime}}\left(z_{2}^{\prime}\right)\right]}=\lambda_{\alpha_{1}^{\prime}}\left(z_{1}^{\prime}\right) e^{i \psi_{\alpha_{1}^{\prime}}\left(z_{1}^{\prime}\right)}, \\
Y_{\left(\alpha_{1}^{\prime} \cap \beta_{1}^{\prime}\right) \times \gamma_{1}^{\prime}}\left(z^{\prime}\right) & =Y_{\alpha_{1}^{\prime}}\left(z_{1}^{\prime}\right) .9
\end{aligned}
$$

Also, equation (2) becomes

$$
\begin{aligned}
& Y_{\left(\alpha_{1}^{\prime} \times \gamma_{1}^{\prime}\right) \cap\left(\beta_{1}^{\prime} \times \gamma_{1}^{\prime}\right)}\left(z^{\prime}\right)=\min \left[\lambda_{\alpha_{1}^{\prime}}\left(z_{1}^{\prime}\right), \lambda_{\gamma_{1}^{\prime}}\left(z_{2}^{\prime}\right)\right] e^{i \min \left[\psi_{\alpha_{1}^{\prime}}\left(z_{1}^{\prime}\right), \psi_{\gamma_{1}^{\prime}}\left(z_{2}^{\prime}\right)\right]}=\lambda_{\alpha_{1}^{\prime}}\left(z_{1}^{\prime}\right) e^{i \psi_{\alpha_{1}^{\prime}}\left(z_{1}^{\prime}\right)}, \\
& Y_{\left(\alpha_{1}^{\prime} \times \gamma_{1}^{\prime}\right) \cap\left(\beta_{1}^{\prime} \times \gamma_{1}^{\prime}\right)}\left(z^{\prime}\right)=Y_{\alpha_{1}^{\prime}}\left(z_{1}^{\prime}\right) .
\end{aligned}
$$

From (9) and (10), we have

$$
Y_{\left(\alpha_{1}^{\prime} \cap \beta_{1}^{\prime}\right) \times \gamma_{1}^{\prime}}\left(z^{\prime}\right)=Y_{\left(\alpha_{1}^{\prime} \times \gamma_{1}^{\prime}\right) \cap\left(\beta_{1}^{\prime} \times \gamma_{1}^{\prime}\right)}\left(z^{\prime}\right),
$$

Case V. If

$$
\lambda_{\beta_{1}^{\prime}}\left(z_{1}^{\prime}\right) \leq \lambda_{\alpha_{1}^{\prime}}\left(z_{1}^{\prime}\right) \leq \lambda_{\gamma_{1}^{\prime}}\left(z_{2}^{\prime}\right) \text { and } \psi_{\beta_{1}^{\prime}}\left(z_{1}^{\prime}\right) \leq \psi_{\alpha_{1}^{\prime}}\left(z_{1}^{\prime}\right) \leq \psi_{\gamma_{1}^{\prime}}\left(z_{2}^{\prime}\right) .
$$

then equation (1) becomes

$$
\begin{aligned}
Y_{\left(\alpha_{1}^{\prime} \cap \beta_{1}^{\prime}\right) \times \gamma_{1}^{\prime}}\left(z^{\prime}\right) & =\min \left[\lambda_{\beta_{1}^{\prime}}\left(z_{1}^{\prime}\right), \lambda_{\gamma_{1}^{\prime}}\left(z_{2}^{\prime}\right)\right] e^{i \min \left[\psi_{\beta_{1}^{\prime}}\left(z_{1}^{\prime}\right), \psi_{\gamma_{1}^{\prime}}\left(z_{2}^{\prime}\right)\right]}=\lambda_{\beta_{1}^{\prime}}\left(z_{1}^{\prime}\right) e^{i \psi_{\beta_{1}^{\prime}}\left(z_{1}^{\prime}\right)}, \\
Y_{\left(\alpha_{1}^{\prime} \cap \beta_{1}^{\prime}\right) \times \gamma_{1}^{\prime}}\left(z^{\prime}\right) & =Y_{\beta_{1}^{\prime}}\left(z_{1}^{\prime}\right) .
\end{aligned}
$$

Also, equation (2) becomes

$$
\begin{aligned}
& Y_{\left(\alpha_{1}^{\prime} \times \gamma_{1}^{\prime}\right) \cap\left(\beta_{1}^{\prime} \times \gamma_{1}^{\prime}\right)}\left(z^{\prime}\right)=\min \left[\lambda_{\alpha_{1}^{\prime}}\left(z_{1}^{\prime}\right), \lambda_{\beta_{1}^{\prime}}\left(z_{1}^{\prime}\right)\right] e^{i \min \left[\psi_{\alpha_{1}^{\prime}}\left(z_{1}^{\prime}\right), \psi_{\beta_{1}^{\prime}}\left(z_{1}^{\prime}\right)\right]}=\lambda_{\beta_{1}^{\prime}}\left(z_{1}^{\prime}\right) e^{i \psi_{\beta_{1}^{\prime}}\left(z_{1}^{\prime}\right)}, \\
& Y_{\left(\alpha_{1}^{\prime} \times \gamma_{1}^{\prime}\right) \cap\left(\beta_{1}^{\prime} \times \gamma_{1}^{\prime}\right)}\left(z^{\prime}\right)=Y_{\beta_{1}^{\prime}}\left(z_{1}^{\prime}\right) .12
\end{aligned}
$$

From (11) and (12), we have

$$
Y_{\left(\alpha_{1}^{\prime} \cap \beta_{1}^{\prime}\right) \times \gamma_{1}^{\prime}}\left(z^{\prime}\right)=Y_{\left(\alpha_{1}^{\prime} \times \gamma_{1}^{\prime}\right) \cap\left(\beta_{1}^{\prime} \times \gamma_{1}^{\prime}\right)}\left(z^{\prime}\right) .
$$

Case VI. If

$$
\lambda_{\gamma_{1}^{\prime}}\left(z_{2}^{\prime}\right) \leq \lambda_{\beta_{1}^{\prime}}\left(z_{1}^{\prime}\right) \leq \lambda_{\alpha_{1}^{\prime}}\left(z_{1}^{\prime}\right) \text { and } \psi_{\gamma_{1}^{\prime}}\left(z_{2}^{\prime}\right) \leq \psi_{\beta_{1}^{\prime}}\left(z_{1}^{\prime}\right) \leq \psi_{\alpha_{1}^{\prime}}\left(z_{1}^{\prime}\right) .
$$

then equation (1) becomes

$$
\begin{aligned}
Y_{\left(\alpha_{1}^{\prime} \cap \beta_{1}^{\prime}\right) \times \gamma_{1}^{\prime}}\left(z^{\prime}\right) & =\min \left[\lambda_{\beta_{1}^{\prime}}\left(z_{1}^{\prime}\right), \lambda_{\gamma_{1}^{\prime}}\left(z_{2}^{\prime}\right)\right] e^{i \min \left[\psi_{\beta_{1}^{\prime}}\left(z_{1}^{\prime}\right), \psi_{\gamma_{1}^{\prime}}\left(z_{2}^{\prime}\right)\right]}=\lambda_{\gamma_{1}^{\prime}}\left(z_{2}^{\prime}\right) e^{i \psi_{\gamma_{1}^{\prime}}\left(z_{2}^{\prime}\right)}, \\
Y_{\left(\alpha_{1}^{\prime} \cap \beta_{1}^{\prime}\right) \times \gamma_{1}^{\prime}}\left(z^{\prime}\right) & =Y_{\gamma_{1}^{\prime}}\left(z_{2}^{\prime}\right) .
\end{aligned}
$$

Also, equation (2) becomes

$$
\begin{aligned}
Y_{\left(\alpha_{1}^{\prime} \times \gamma_{1}^{\prime}\right) \cap\left(\beta_{1}^{\prime} \times \gamma_{1}^{\prime}\right)}\left(z^{\prime}\right) & =\min \left[\lambda_{\gamma_{1}^{\prime}}\left(z_{2}^{\prime}\right), \lambda_{\gamma_{1}^{\prime}}\left(z_{2}^{\prime}\right)\right] e^{i \min \left[\psi_{\gamma_{1}^{\prime}}\left(z_{2}^{\prime}\right), \psi_{\gamma_{1}^{\prime}}\left(z_{2}^{\prime}\right)\right]}=\lambda_{\gamma_{1}^{\prime}}\left(z_{2}^{\prime}\right) e^{i \psi_{\gamma_{1}^{\prime}}\left(z_{2}^{\prime}\right)}, \\
Y_{\left(\alpha_{1}^{\prime} \times \gamma_{1}^{\prime}\right) \cap\left(\beta_{1}^{\prime} \times \gamma_{1}^{\prime}\right)}\left(z^{\prime}\right) & =Y_{\gamma_{1}^{\prime}}\left(z_{2}^{\prime}\right) .
\end{aligned}
$$


From (13) and (14), we have

$$
Y_{\left(\alpha_{1}^{\prime} \cap \beta_{1}^{\prime}\right) \times \gamma_{1}^{\prime}}\left(z^{\prime}\right)=Y_{\left(\alpha_{1}^{\prime} \times \gamma_{1}^{\prime}\right) \cap\left(\beta_{1}^{\prime} \times \gamma_{1}^{\prime}\right)}\left(z^{\prime}\right) .
$$

Thus from all above cases, we have

$$
\left(\alpha_{1}^{\prime} \cap \beta_{1}^{\prime}\right) \times \gamma_{1}^{\prime}=\left(\alpha_{1}^{\prime} \times \gamma_{1}^{\prime}\right) \cap\left(\beta_{1}^{\prime} \times \gamma_{1}^{\prime}\right) .
$$

Example 4.4. Suppose

$$
\begin{aligned}
\alpha_{1}^{\prime} & =\frac{0.1 e^{i 1.2 \pi}}{-1}+\frac{0.3 e^{i 2 \pi}}{-2}+\frac{0.9 e^{i \pi}}{-3}, \\
\beta_{1}^{\prime} & =\frac{0.4 e^{i 1.4 \pi}}{-1}+\frac{0.6 e^{i 1.2 \pi}}{-2}+\frac{0.2 e^{i 1.6 \pi}}{-3}, \\
\gamma_{1}^{\prime} & =\frac{0.7 e^{i 1.1 \pi}}{-1}+\frac{0.8 e^{i 0.8 \pi}}{-2}+\frac{0.1 e^{i 1.3 \pi}}{-3} .
\end{aligned}
$$

are the three complex fuzzy sets. Using the CF intersection and Cartesian product we will prove the above statement as:

$$
\begin{aligned}
\alpha_{1}^{\prime} \cap \beta_{1}^{\prime}= & \frac{0.1 e^{i 1.2 \pi}}{-1}+\frac{0.3 e^{i 1.2 \pi}}{-2}+\frac{0.2 e^{i \pi}}{-3} \\
\left(\alpha_{1}^{\prime} \cap \beta_{1}^{\prime}\right) \times \gamma_{1}^{\prime}= & \left(\frac{0.1 e^{i 1.2 \pi}}{-1}+\frac{0.3 e^{i 1.2 \pi}}{-2}+\frac{0.2 e^{i \pi}}{-3}\right) \times\left(\frac{0.7 e^{i 1.1 \pi}}{-1}+\frac{0.8 e^{i 0.8 \pi}}{-2}+\frac{0.1 e^{i 1.3 \pi}}{-3}\right) \\
\left(\alpha_{1}^{\prime} \cap \beta_{1}^{\prime}\right) \times \gamma_{1}^{\prime}= & \left\{\frac{0.1 e^{i 1.1 \pi}}{(-1,-1)}+\frac{0.1 e^{i 0.8 \pi}}{(-1,-2)}+\frac{0.1 e^{i 1.2 \pi}}{(-1,-3)}+\right. \\
& \frac{0.3 e^{i 1.1 \pi}}{(-2,-1)}+\frac{0.3 e^{i 0.8 \pi}}{(-2,-2)}+\frac{0.1 e^{i 1.2 \pi}}{(-2,-3)}+ \\
& \left.\frac{0.2 e^{i \pi}}{(-3,-1)}+\frac{0.2 e^{i 0.8 \pi}}{(-3,-2)}+\frac{0.1 e^{i \pi}}{(-3,-3)}\right\}
\end{aligned}
$$

Now,

$$
\begin{aligned}
\alpha_{1}^{\prime} \times \gamma_{1}^{\prime}= & \left(\frac{0.1 e^{i 1.2 \pi}}{-1}+\frac{0.3 e^{i 2 \pi}}{-2}+\frac{0.9 e^{i \pi}}{-3}\right) \times\left(\frac{0.7 e^{i 1.1 \pi}}{-1}+\frac{0.8 e^{i 0.8 \pi}}{-2}+\frac{0.1 e^{i 1.3 \pi}}{-3}\right) \\
= & \left\{\frac{0.1 e^{i 1.1 \pi}}{(-1,-1)}+\frac{0.1 e^{i 0.8 \pi}}{(-1,-2)}+\frac{0.1 e^{i 1.2 \pi}}{(-1,-3)}+\frac{0.3 e^{i 1.1 \pi}}{(-2,-1)}+\frac{0.3 e^{i 0.8 \pi}}{(-2,-2)}+\frac{0.1 e^{i 1.3 \pi}}{(-2,-3)}+\right. \\
& \left.\frac{0.7 e^{i \pi}}{(-3,-1)}+\frac{0.8 e^{i 0.8 \pi}}{(-3,-2)}+\frac{0.1 e^{i \pi}}{(-3,-3)}\right\}, \\
\beta_{1}^{\prime} \times \gamma_{1}^{\prime}= & \left(\frac{0.4 e^{i 1.4 \pi}}{-1}+\frac{0.6 e^{i 1.2 \pi}}{-2}+\frac{0.2 e^{i 1.6 \pi}}{-3}\right) \times\left(\frac{0.7 e^{i 1.1 \pi}}{-1}+\frac{0.8 e^{i 0.8 \pi}}{-2}+\frac{0.1 e^{i 1.3 \pi}}{-3}\right) \\
= & \left\{\frac{0.4 e^{i 1.1 \pi}}{(-1,-1)}+\frac{0.4 e^{i 0.8 \pi}}{(-1,-2)}+\frac{0.1 e^{i 1.3 \pi}}{(-1,-3)}+\frac{0.6 e^{i 1.1 \pi}}{(-2,-1)}+\frac{0.6 e^{i 0.8 \pi}}{(-2,-2)}+\frac{0.1 e^{i 1.2 \pi}}{(-2,-3)}+\right. \\
& \left.\frac{0.2 e^{i 1.1 \pi}}{(-3,-1)}+\frac{0.2 e^{i 0.8 \pi}}{(-3,-2)}+\frac{0.1 e^{i 1.3 \pi}}{(-3,-3)}\right\},
\end{aligned}
$$




$$
\begin{aligned}
\left(\alpha_{1}^{\prime} \times \gamma_{1}^{\prime}\right) \cap\left(\beta_{1}^{\prime} \times \gamma_{1}^{\prime}\right)= & \left\{\frac{0.1 e^{i 1.1 \pi}}{(-1,-1)}+\frac{0.1 e^{i 0.8 \pi}}{(-1,-2)}+\frac{0.1 e^{i 1.2 \pi}}{(-1,-3)}+2 \frac{0.3 e^{i 1.1 \pi}}{(-2,-1)}+\frac{0.3 e^{i 0.8 \pi}}{(-2,-2)}+\frac{0.1 e^{i 1.2 \pi}}{(-2,-3)}+\right. \\
& \left.\frac{0.2 e^{i \pi}}{(-3,-1)}+\frac{0.2 e^{i 0.8 \pi}}{(-3,-2)}+\frac{0.1 e^{i \pi}}{(-3,-3)}\right\} .
\end{aligned}
$$

From (1) and (2), we have

$$
\left(\alpha_{1}^{\prime} \cap \beta_{1}^{\prime}\right) \times \gamma_{1}^{\prime}=\left(\alpha_{1}^{\prime} \times \gamma_{1}^{\prime}\right) \cap\left(\beta_{1}^{\prime} \times \gamma_{1}^{\prime}\right) .
$$

Proposition 4.5. If $\alpha_{1}^{\prime}$ and $\beta_{1}^{\prime}$ are two complex fuzzy sets on the universal set $U$, then

$$
\bar{\alpha}_{1}^{\prime} \ominus \bar{\beta}_{1}^{\prime}=\beta_{1}^{\prime} \ominus \alpha_{1}^{\prime} \text {. }
$$

Proof. Let $Y_{\alpha_{1}^{\prime}}\left(z^{\prime}\right)=\lambda_{\alpha_{1}^{\prime}}\left(z^{\prime}\right) e^{i \psi_{\alpha_{1}^{\prime}}\left(z^{\prime}\right)}$ and $Y_{\beta_{1}^{\prime}}\left(z^{\prime}\right)=\lambda_{\beta_{1}^{\prime}}\left(z^{\prime}\right) e^{i \psi_{\beta_{1}^{\prime}}\left(z^{\prime}\right)}$ be the membership functions of $\alpha_{1}^{\prime}$ and $\beta_{1}^{\prime}$. Using the definition of standard complement and bounded difference we will prove the following statement,

$$
Y_{\bar{\alpha}_{1}^{\prime} \ominus \bar{\beta}_{1}^{\prime}}\left(z^{\prime}\right)=Y_{\beta_{1}^{\prime} \ominus \alpha_{1}^{\prime}}\left(z^{\prime}\right)
$$

As

$$
\begin{aligned}
Y_{\bar{\alpha}_{1}^{\prime} \ominus \bar{\beta}_{1}^{\prime}}\left(z^{\prime}\right) & =\lambda_{\bar{\alpha}_{1}^{\prime} \ominus \bar{\beta}_{1}^{\prime}}\left(z^{\prime}\right) e^{i \psi_{\bar{\alpha}_{1}^{\prime} \ominus \bar{\beta}_{1}^{\prime}}\left(z^{\prime}\right)} \\
& =\max \left[0, \lambda_{\bar{\alpha}_{1}^{\prime}}\left(z^{\prime}\right)-\lambda_{\bar{\beta}_{1}^{\prime}}\left(z^{\prime}\right)\right] e^{i \max \left[0, \psi_{\bar{\alpha}_{1}}\left(z^{\prime}\right)-\psi_{\bar{\beta}_{1}^{\prime}}\left(z^{\prime}\right)\right]} \\
& =\max \left[0,1-\lambda_{\alpha_{1}^{\prime}}\left(z^{\prime}\right)-\left[1-\lambda_{\beta_{1}^{\prime}}\left(z^{\prime}\right)\right]\right] e^{i \max \left[0,1-\psi_{\alpha_{1}^{\prime}}\left(z^{\prime}\right)-\left[1-\psi_{\beta_{1}^{\prime}}\left(z^{\prime}\right)\right]\right]} \\
& =\max \left[0,1-\lambda_{\alpha_{1}^{\prime}}\left(z^{\prime}\right)-1+\lambda_{\beta_{1}^{\prime}}\left(z^{\prime}\right)\right] e^{i \max \left[0,1-\psi_{\alpha_{1}^{\prime}}\left(z^{\prime}\right)-1+\psi_{\beta_{1}^{\prime}}\left(z^{\prime}\right)\right]} \\
& =\max \left[0, \lambda_{\beta_{1}^{\prime}}\left(z^{\prime}\right)-\lambda_{\alpha_{1}^{\prime}}\left(z^{\prime}\right)\right] e^{i \max \left[0, \psi_{\beta_{1}^{\prime}}\left(z^{\prime}\right)-\psi_{\alpha_{1}^{\prime}}\left(z^{\prime}\right)\right]} \\
& =\lambda_{\beta_{1}^{\prime} \ominus \alpha_{1}^{\prime}}\left(z^{\prime}\right) e^{i \psi_{\beta_{1}^{\prime} \ominus \alpha_{1}^{\prime}}\left(z^{\prime}\right)} \\
Y_{\bar{\alpha}_{1}^{\prime} \ominus \bar{\beta}_{1}^{\prime}}\left(z^{\prime}\right) & =Y_{\beta_{1}^{\prime} \ominus \alpha_{1}^{\prime}}\left(z^{\prime}\right) .
\end{aligned}
$$

Therefore,

$$
\bar{\alpha}_{1}^{\prime} \ominus \bar{\beta}_{1}^{\prime}=\beta_{1}^{\prime} \ominus \alpha_{1}^{\prime} .
$$

Proposition 4.6. If $\alpha_{1}^{\prime}$ and $\beta_{1}^{\prime}$ are two complex fuzzy sets on the universe of discourse $U$, then

$$
\left(\alpha_{1}^{\prime} \cup \beta_{1}^{\prime}\right) \ominus\left(\alpha_{1}^{\prime} \cap \beta_{1}^{\prime}\right)=\left(\alpha_{1}^{\prime} \ominus \beta_{1}^{\prime}\right) \cup\left(\beta_{1}^{\prime} \ominus \alpha_{1}^{\prime}\right) .
$$

Proof. Let $Y_{\alpha_{1}^{\prime}}\left(z^{\prime}\right)=\lambda_{\alpha_{1}^{\prime}}\left(z^{\prime}\right) e^{i \psi_{\alpha_{1}^{\prime}}\left(z^{\prime}\right)}$ and $Y_{\beta_{1}^{\prime}}\left(z^{\prime}\right)=\lambda_{\beta_{1}^{\prime}}\left(z^{\prime}\right) e^{i \psi_{\beta_{1}^{\prime}}\left(z^{\prime}\right)}$ be the membership functions of $\alpha_{1}^{\prime}$ and $\beta_{1}^{\prime}$. Using the definition of complex fuzzy union, bounded difference and complex fuzzy intersection we will prove the following statement

$$
Y_{\left(\alpha_{1}^{\prime} \cup \beta_{1}^{\prime}\right) \ominus\left(\alpha_{1}^{\prime} \cap \beta_{1}^{\prime}\right)}\left(z^{\prime}\right)=Y_{\left(\alpha_{1}^{\prime} \ominus \beta_{1}^{\prime}\right) \cup\left(\beta_{1}^{\prime} \ominus \alpha_{1}^{\prime}\right)}\left(z^{\prime}\right) .
$$

The L.H.S of the above statement is given as:

$$
\begin{aligned}
Y_{\left(\alpha_{1}^{\prime} \cup \beta_{1}^{\prime}\right) \ominus\left(\alpha_{1}^{\prime} \cap \beta_{1}^{\prime}\right)}\left(z^{\prime}\right)= & \lambda_{\left(\alpha_{1}^{\prime} \cup \beta_{1}^{\prime}\right) \ominus\left(\alpha_{1}^{\prime} \cap \beta_{1}^{\prime}\right)}\left(z^{\prime}\right) e^{i \psi_{\left(\alpha_{1}^{\prime} \cup \beta_{1}^{\prime}\right) \ominus\left(\alpha_{1}^{\prime} \cap \beta_{1}^{\prime}\right)}\left(z^{\prime}\right)} \\
= & \max \left[0, \lambda_{\alpha_{1}^{\prime} \cup \beta_{1}^{\prime}}\left(z^{\prime}\right)-\lambda_{\alpha_{1}^{\prime} \cap \beta_{1}^{\prime}}\left(z^{\prime}\right)\right] e^{i \max \left[0, \psi_{\alpha_{1}^{\prime} \cup \beta_{1}^{\prime}}\left(z^{\prime}\right)-\psi_{\alpha_{1}^{\prime} \cap \beta_{1}^{\prime}}\left(z^{\prime}\right)\right]}, \\
Y_{\left(\alpha_{1}^{\prime} \cup \beta_{1}^{\prime}\right) \ominus\left(\alpha_{1}^{\prime} \cap \beta_{1}^{\prime}\right)}\left(z^{\prime}\right)= & \max \left[0, \max \left[\lambda_{\alpha_{1}^{\prime}}\left(z^{\prime}\right), \lambda_{\beta_{1}^{\prime}}\left(z^{\prime}\right)\right]\right. \\
& \left.\quad-1 \min \left[\lambda_{\alpha_{1}^{\prime}}\left(z^{\prime}\right), \lambda_{\beta_{1}^{\prime}}\left(z^{\prime}\right)\right]\right] e^{i \max \left[0, \max \left[\psi_{\alpha_{1}^{\prime}}\left(z^{\prime}\right), \psi_{\beta_{1}^{\prime}}\left(z^{\prime}\right)\right]-\min \left[\psi_{\alpha_{1}^{\prime}}\left(z^{\prime}\right), \psi_{\beta_{1}^{\prime}}\left(z^{\prime}\right)\right]\right]}
\end{aligned}
$$


The R.H.S is given as:

$$
\begin{aligned}
Y_{\left(\alpha_{1}^{\prime} \ominus \beta_{1}^{\prime}\right) \cup\left(\beta_{1}^{\prime} \ominus \alpha_{1}^{\prime}\right)}\left(z^{\prime}\right)= & \lambda_{\left(\alpha_{1}^{\prime} \ominus \beta_{1}^{\prime}\right) \cup\left(\beta_{1}^{\prime} \ominus \alpha_{1}^{\prime}\right)}\left(z^{\prime}\right) e^{i \psi_{\left(\alpha_{1}^{\prime} \ominus \beta_{1}^{\prime}\right) \cup\left(\beta_{1}^{\prime} \ominus \alpha_{1}^{\prime}\right)}\left(z^{\prime}\right)} \\
= & \max \left[\lambda_{\alpha_{1}^{\prime} \ominus \beta_{1}^{\prime}}\left(z^{\prime}\right), \lambda_{\beta_{1}^{\prime} \ominus \alpha_{1}^{\prime}}\left(z^{\prime}\right)\right] e^{i \max \left[\psi_{\alpha_{1}^{\prime} \ominus \beta_{1}^{\prime}}\left(z^{\prime}\right), \psi_{\beta_{1}^{\prime} \ominus \alpha_{1}^{\prime}}\left(z^{\prime}\right)\right]} \\
Y_{\left(\alpha_{1}^{\prime} \ominus \beta_{1}^{\prime}\right) \cup\left(\beta_{1}^{\prime} \ominus \alpha_{1}^{\prime}\right)}\left(z^{\prime}\right)= & \max \left[\max \left[0, \lambda_{\alpha_{1}^{\prime}}\left(z^{\prime}\right)-\lambda_{\beta_{1}^{\prime}}\left(z^{\prime}\right)\right], \max \left[0, \lambda_{\beta_{1}^{\prime}}\left(z^{\prime}\right)-\lambda_{\alpha_{1}^{\prime}}\left(z^{\prime}\right)\right]\right] \\
& e^{i \max \left[\max \left[0, \psi_{\alpha_{1}^{\prime}}\left(z^{\prime}\right)-\psi_{\beta_{1}^{\prime}}\left(z^{\prime}\right)\right], \max \left[0, \psi_{\beta_{1}^{\prime}}\left(z^{\prime}\right)-\psi_{\alpha_{1}^{\prime}}\left(z^{\prime}\right)\right]\right]} .
\end{aligned}
$$

To prove the above statement, we have the following cases:

Case I. If

$$
\lambda_{\alpha_{1}^{\prime}}\left(z^{\prime}\right) \leq \lambda_{\beta_{1}^{\prime}}\left(z^{\prime}\right) \text { and } \psi_{\alpha_{1}^{\prime}}\left(z^{\prime}\right) \leq \psi_{\beta_{1}^{\prime}}\left(z^{\prime}\right),
$$

then equation (1) becomes

$$
\begin{aligned}
Y_{\left(\alpha_{1}^{\prime} \cup \beta_{1}^{\prime}\right) \ominus\left(\alpha_{1}^{\prime} \cap \beta_{1}^{\prime}\right)}\left(z^{\prime}\right) & =\max \left[0, \lambda_{\beta_{1}^{\prime}}\left(z^{\prime}\right)-\lambda_{\alpha_{1}^{\prime}}\left(z^{\prime}\right)\right] e^{i \max \left[0, \psi_{\beta_{1}^{\prime}}\left(z^{\prime}\right)-\psi_{\alpha_{1}^{\prime}}\left(z^{\prime}\right)\right]} \\
Y_{\left(\alpha_{1}^{\prime} \cup \beta_{1}^{\prime}\right) \ominus\left(\alpha_{1}^{\prime} \cap \beta_{1}^{\prime}\right)}\left(z^{\prime}\right) & =\left[\lambda_{\beta_{1}^{\prime}}\left(z^{\prime}\right)-\lambda_{\alpha_{1}^{\prime}}\left(z^{\prime}\right)\right] e^{i\left[\psi_{\beta_{1}^{\prime}}\left(z^{\prime}\right)-\psi_{\alpha_{1}^{\prime}}\left(z^{\prime}\right)\right]} .
\end{aligned}
$$

Also, equation (2) becomes

$$
\begin{aligned}
Y_{\left(\alpha_{1}^{\prime} \ominus \beta_{1}^{\prime}\right) \cup\left(\beta_{1}^{\prime} \ominus \alpha_{1}^{\prime}\right)}\left(z^{\prime}\right) & =\max \left[0, \lambda_{\beta_{1}^{\prime}}\left(z^{\prime}\right)-\lambda_{\alpha_{1}^{\prime}}\left(z^{\prime}\right)\right] e^{i \max \left[0, \psi_{\beta_{1}^{\prime}}\left(z^{\prime}\right)-\psi_{\alpha_{1}^{\prime}}\left(z^{\prime}\right)\right]} \\
Y_{\left(\alpha_{1}^{\prime} \ominus \beta_{1}^{\prime}\right) \cup\left(\beta_{1}^{\prime} \ominus \alpha_{1}^{\prime}\right)}\left(z^{\prime}\right) & =\left[\lambda_{\beta_{1}^{\prime}}\left(z^{\prime}\right)-\lambda_{\alpha_{1}^{\prime}}\left(z^{\prime}\right)\right] e^{i\left[\psi_{\beta_{1}^{\prime}}\left(z^{\prime}\right)-\psi_{\alpha_{1}^{\prime}}\left(z^{\prime}\right)\right]} .
\end{aligned}
$$

From (3)and (4), we get

$$
Y_{\left(\alpha_{1}^{\prime} \cup \beta_{1}^{\prime}\right) \ominus\left(\alpha_{1}^{\prime} \cap \beta_{1}^{\prime}\right)}\left(z^{\prime}\right)=Y_{\left(\alpha_{1}^{\prime} \ominus \beta_{1}^{\prime}\right) \cup\left(\beta_{1}^{\prime} \ominus \alpha_{1}^{\prime}\right)}\left(z^{\prime}\right) .
$$

Case II. If

$$
\lambda_{\alpha_{1}^{\prime}}\left(z^{\prime}\right) \geq \lambda_{\beta_{1}^{\prime}}\left(z^{\prime}\right) \text { and } \psi_{\alpha_{1}^{\prime}}\left(z^{\prime}\right) \geq \psi_{\beta_{1}^{\prime}}\left(z^{\prime}\right)
$$

then equation (1) becomes

$$
\begin{aligned}
Y_{\left(\alpha_{1}^{\prime} \cup \beta_{1}^{\prime}\right) \ominus\left(\alpha_{1}^{\prime} \cap \beta_{1}^{\prime}\right)}\left(z^{\prime}\right) & =\max \left[0, \lambda_{\alpha_{1}^{\prime}}\left(z^{\prime}\right)-\lambda_{\beta_{1}^{\prime}}\left(z^{\prime}\right)\right] e^{i \max \left[0, \psi_{\alpha_{1}^{\prime}}\left(z^{\prime}\right)-\psi_{\beta_{1}^{\prime}}\left(z^{\prime}\right)\right]} \\
Y_{\left(\alpha_{1}^{\prime} \cup \beta_{1}^{\prime}\right) \ominus\left(\alpha_{1}^{\prime} \cap \beta_{1}^{\prime}\right)}\left(z^{\prime}\right) & =\left[\lambda_{\alpha_{1}^{\prime}}\left(z^{\prime}\right)-\lambda_{\beta_{1}^{\prime}}\left(z^{\prime}\right)\right] e^{i\left[\psi_{\alpha_{1}^{\prime}}\left(z^{\prime}\right)-\psi_{\beta_{1}^{\prime}}\left(z^{\prime}\right)\right]} .
\end{aligned}
$$

Also, equation (2) becomes

$$
\begin{aligned}
Y_{\left(\alpha_{1}^{\prime} \ominus \beta_{1}^{\prime}\right) \cup\left(\beta_{1}^{\prime} \ominus \alpha_{1}^{\prime}\right)}\left(z^{\prime}\right) & =\max \left[\lambda_{\alpha_{1}^{\prime}}\left(z^{\prime}\right)-\lambda_{\beta_{1}^{\prime}}\left(z^{\prime}\right), 0\right] e^{i \max \left[\psi_{\alpha_{1}^{\prime}}\left(z^{\prime}\right)-\psi_{\beta_{1}^{\prime}}\left(z^{\prime}\right), 0\right]} \\
Y_{\left(\alpha_{1}^{\prime} \ominus \beta_{1}^{\prime}\right) \cup\left(\beta_{1}^{\prime} \ominus \alpha_{1}^{\prime}\right)}\left(z^{\prime}\right) & =\left[\lambda_{\alpha_{1}^{\prime}}\left(z^{\prime}\right)-\lambda_{\beta_{1}^{\prime}}\left(z^{\prime}\right)\right] e^{i\left[\psi_{\alpha_{1}^{\prime}}\left(z^{\prime}\right)-\psi_{\beta_{1}^{\prime}}\left(z^{\prime}\right)\right]} .
\end{aligned}
$$

From (5)and (6), we get

$$
Y_{\left(\alpha_{1}^{\prime} \cup \beta_{1}^{\prime}\right) \ominus\left(\alpha_{1}^{\prime} \cap \beta_{1}^{\prime}\right)}\left(z^{\prime}\right)=Y_{\left(\alpha_{1}^{\prime} \ominus \beta_{1}^{\prime}\right) \cup\left(\beta_{1}^{\prime} \ominus \alpha_{1}^{\prime}\right)}\left(z^{\prime}\right) .
$$

Case III. If

$$
\lambda_{\alpha_{1}^{\prime}}\left(z^{\prime}\right) \leq \lambda_{\beta_{1}^{\prime}}\left(z^{\prime}\right) \text { and } \psi_{\alpha_{1}^{\prime}}\left(z^{\prime}\right) \geq \psi_{\beta_{1}^{\prime}}\left(z^{\prime}\right)
$$


then equation (1) becomes

$$
\begin{aligned}
Y_{\left(\alpha_{1}^{\prime} \cup \beta_{1}^{\prime}\right) \ominus\left(\alpha_{1}^{\prime} \cap \beta_{1}^{\prime}\right)}\left(z^{\prime}\right) & =\max \left[0, \lambda_{\beta_{1}^{\prime}}\left(z^{\prime}\right)-\lambda_{\alpha_{1}^{\prime}}\left(z^{\prime}\right)\right] e^{i \max \left[0, \psi_{\alpha_{1}^{\prime}}\left(z^{\prime}\right)-\psi_{\beta_{1}^{\prime}}\left(z^{\prime}\right)\right]}, \\
Y_{\left(\alpha_{1}^{\prime} \cup \beta_{1}^{\prime}\right) \ominus\left(\alpha_{1}^{\prime} \cap \beta_{1}^{\prime}\right)}\left(z^{\prime}\right) & =\left[\lambda_{\beta_{1}^{\prime}}\left(z^{\prime}\right)-\lambda_{\alpha_{1}^{\prime}}\left(z^{\prime}\right)\right] e^{i\left[\psi_{\alpha_{1}^{\prime}}\left(z^{\prime}\right)-\psi_{\beta_{1}^{\prime}}\left(z^{\prime}\right)\right]} .
\end{aligned}
$$

Also, equation (2) becomes

$$
\begin{aligned}
& Y_{\left(\alpha_{1}^{\prime} \ominus \beta_{1}^{\prime}\right) \cup\left(\beta_{1}^{\prime} \ominus \alpha_{1}^{\prime}\right)}\left(z^{\prime}\right)=\max \left[0, \lambda_{\beta_{1}^{\prime}}\left(z^{\prime}\right)-\lambda_{\alpha_{1}^{\prime}}\left(z^{\prime}\right)\right] e^{i \max \left[\psi_{\alpha_{1}^{\prime}}\left(z^{\prime}\right)-\psi_{\beta_{1}^{\prime}}\left(z^{\prime}\right), 0\right]}, \\
& Y_{\left(\alpha_{1}^{\prime} \ominus \beta_{1}^{\prime}\right) \cup\left(\beta_{1}^{\prime} \ominus \alpha_{1}^{\prime}\right)}\left(z^{\prime}\right)=\left[\lambda_{\beta_{1}^{\prime}}\left(z^{\prime}\right)-\lambda_{\alpha_{1}^{\prime}}\left(z^{\prime}\right)\right] e^{i\left[\psi_{\alpha_{1}^{\prime}}\left(z^{\prime}\right)-\psi_{\beta_{1}^{\prime}}\left(z^{\prime}\right)\right]} .
\end{aligned}
$$

From (7)and (8), we get

$$
Y_{\left(\alpha_{1}^{\prime} \cup \beta_{1}^{\prime}\right) \ominus\left(\alpha_{1}^{\prime} \cap \beta_{1}^{\prime}\right)}\left(z^{\prime}\right)=Y_{\left(\alpha_{1}^{\prime} \ominus \beta_{1}^{\prime}\right) \cup\left(\beta_{1}^{\prime} \ominus \alpha_{1}^{\prime}\right)}\left(z^{\prime}\right) .
$$

Case IV. If

$$
\lambda_{\alpha_{1}^{\prime}}\left(z^{\prime}\right) \geq \lambda_{\beta_{1}^{\prime}}\left(z^{\prime}\right) \text { and } \psi_{\alpha_{1}^{\prime}}\left(z^{\prime}\right) \leq \psi_{\beta_{1}^{\prime}}\left(z^{\prime}\right),
$$

then equation (1) becomes

$$
\begin{aligned}
Y_{\left(\alpha_{1}^{\prime} \cup \beta_{1}^{\prime}\right) \ominus\left(\alpha_{1}^{\prime} \cap \beta_{1}^{\prime}\right)}\left(z^{\prime}\right) & =\max \left[0, \lambda_{\alpha_{1}^{\prime}}\left(z^{\prime}\right)-\lambda_{\beta_{1}^{\prime}}\left(z^{\prime}\right)\right] e^{i \max \left[0, \psi_{\beta_{1}^{\prime}}\left(z^{\prime}\right)-\psi_{\alpha_{1}^{\prime}}\left(z^{\prime}\right)\right]}, \\
Y_{\left(\alpha_{1}^{\prime} \cup \beta_{1}^{\prime}\right) \ominus\left(\alpha_{1}^{\prime} \cap \beta_{1}^{\prime}\right)}\left(z^{\prime}\right) & =\left[\lambda_{\alpha_{1}^{\prime}}\left(z^{\prime}\right)-\lambda_{\beta_{1}^{\prime}}\left(z^{\prime}\right)\right] e^{i\left[\psi_{\beta_{1}^{\prime}}\left(z^{\prime}\right)-\psi_{\alpha_{1}^{\prime}}\left(z^{\prime}\right)\right]} .
\end{aligned}
$$

Also, equation (2) becomes

$$
\begin{aligned}
& Y_{\left(\alpha_{1}^{\prime} \ominus \beta_{1}^{\prime}\right) \cup\left(\beta_{1}^{\prime} \ominus \alpha_{1}^{\prime}\right)}\left(z^{\prime}\right)=\max \left[\lambda_{\alpha_{1}^{\prime}}\left(z^{\prime}\right)-\lambda_{\beta_{1}^{\prime}}\left(z^{\prime}\right), 0\right] e^{i \max \left[0, \psi_{\beta_{1}^{\prime}}\left(z^{\prime}\right)-\psi_{\alpha_{1}^{\prime}}\left(z^{\prime}\right)\right]}, \\
& Y_{\left(\alpha_{1}^{\prime} \ominus \beta_{1}^{\prime}\right) \cup\left(\beta_{1}^{\prime} \ominus \alpha_{1}^{\prime}\right)}\left(z^{\prime}\right)=\left[\lambda_{\alpha_{1}^{\prime}}\left(z^{\prime}\right)-\lambda_{\beta_{1}^{\prime}}\left(z^{\prime}\right)\right] e^{i\left[\psi_{\beta_{1}^{\prime}}\left(z^{\prime}\right)-\psi_{\alpha_{1}^{\prime}}\left(z^{\prime}\right)\right]} .
\end{aligned}
$$

From (9)and (10), we get

$$
Y_{\left(\alpha_{1}^{\prime} \cup \beta_{1}^{\prime}\right) \ominus\left(\alpha_{1}^{\prime} \cap \beta_{1}^{\prime}\right)}\left(z^{\prime}\right)=Y_{\left(\alpha_{1}^{\prime} \ominus \beta_{1}^{\prime}\right) \cup\left(\beta_{1}^{\prime} \ominus \alpha_{1}^{\prime}\right)}\left(z^{\prime}\right) .
$$

Thus from the above cases, we have

$$
\left(\alpha_{1}^{\prime} \cup \beta_{1}^{\prime}\right) \ominus\left(\alpha_{1}^{\prime} \cap \beta_{1}^{\prime}\right)=\left(\alpha_{1}^{\prime} \ominus \beta_{1}^{\prime}\right) \cup\left(\beta_{1}^{\prime} \ominus \alpha_{1}^{\prime}\right) .
$$

Example 4.7. Suppose

$$
\begin{aligned}
\alpha_{1}^{\prime} & =\frac{0.3 e^{i 1.2 \pi}}{-1}+\frac{0.6 e^{i \pi}}{-2}+\frac{0.1 e^{i 2 \pi}}{-3} \\
\beta_{1}^{\prime} & =\frac{0.4 e^{i 1.6 \pi}}{-1}+\frac{0.9 e^{i 1.4 \pi}}{-2}+\frac{0.2 e^{i 1.2 \pi}}{-3}
\end{aligned}
$$

are two complex fuzzy sets. Using CF union, bounded product and CF intersection we will prove the above statement as:

$$
\begin{aligned}
\alpha_{1}^{\prime} \cup \beta_{1}^{\prime} & =\frac{0.4 e^{i 1.6 \pi}}{-1}+\frac{0.9 e^{i 1.4 \pi}}{-2}+\frac{0.2 e^{i 2 \pi}}{-3} \\
\alpha_{1}^{\prime} \cap \beta_{1}^{\prime} & =\frac{0.3 e^{i 1.2 \pi}}{-1}+\frac{0.6 e^{i \pi}}{-2}+\frac{0.1 e^{i 1.2 \pi}}{-3}
\end{aligned}
$$




$$
\left(\alpha_{1}^{\prime} \cup \beta_{1}^{\prime}\right) \ominus\left(\alpha_{1}^{\prime} \cap \beta_{1}^{\prime}\right)=\frac{0.1 e^{i 0.4 \pi}}{-1}+\frac{0.3 e^{i 0.4 \pi}}{-2}+\frac{0.1 e^{i 0.8 \pi}}{-3}
$$

Now

$$
\begin{aligned}
& \alpha_{1}^{\prime} \ominus \beta_{1}^{\prime}=\frac{0 e^{i 0 \pi}}{-1}+\frac{0 e^{i 0 \pi}}{-2}+\frac{0 e^{i 0.8 \pi}}{-3} \\
& \beta_{1}^{\prime} \ominus \alpha_{1}^{\prime}=\frac{0.1 e^{i 0.4 \pi}}{-1}+\frac{0.3 e^{i 0.4 \pi}}{-2}+\frac{0.1 e^{i 0 \pi}}{-3}, \\
&\left(\alpha_{1}^{\prime} \ominus \beta_{1}^{\prime}\right) \cup\left(\beta_{1}^{\prime} \ominus \alpha_{1}^{\prime}\right)=\frac{0.1 e^{i 0.4 \pi}}{-1}+\frac{0.3 e^{i 0.4 \pi}}{-2}+\frac{0.1 e^{i 0.8 \pi}}{-3} .
\end{aligned}
$$

From (1) and (2), we have

$$
\left(\alpha_{1}^{\prime} \cup \beta_{1}^{\prime}\right) \ominus\left(\alpha_{1}^{\prime} \cap \beta_{1}^{\prime}\right)=\left(\alpha_{1}^{\prime} \ominus \beta_{1}^{\prime}\right) \cup\left(\beta_{1}^{\prime} \ominus \alpha_{1}^{\prime}\right) .
$$

Proposition 4.8. If $\alpha_{1}^{\prime}$ and $\beta_{1}^{\prime}$ are two complex fuzzy sets on the universe of discourse $U$, then

$$
\overline{\alpha_{1}^{\prime} \cdot \beta_{1}^{\prime}}=\bar{\alpha}_{1}^{\prime} \dot{+} \bar{\beta}_{1}^{\prime} \text {. }
$$

Proof. Let $Y_{\alpha_{1}^{\prime}}\left(z^{\prime}\right)=\lambda_{\alpha_{1}^{\prime}}\left(z^{\prime}\right) e^{i \psi_{\alpha_{1}^{\prime}}\left(z^{\prime}\right)}$ and $Y_{\beta_{1}^{\prime}}\left(z^{\prime}\right)=\lambda_{\beta_{1}^{\prime}}\left(z^{\prime}\right) e^{i \psi_{\beta_{1}^{\prime}}\left(z^{\prime}\right)}$ be the membership functions of $\alpha_{1}^{\prime}$ and $\beta_{1}^{\prime}$. Using the definition of standard $\mathrm{CF}$ complement, dot product and algebraic sum we will prove the following statement

$$
Y_{\overline{\alpha_{1}^{\prime} \cdot \beta_{1}^{\prime}}}\left(z^{\prime}\right)=Y_{\bar{\alpha}_{1}^{\prime}+\bar{\beta}_{1}^{\prime}}\left(z^{\prime}\right)
$$

The L.H.S is given as:

$$
\begin{aligned}
Y_{\overline{\alpha_{1}^{\prime} \cdot \beta_{1}^{\prime}}}\left(z^{\prime}\right) & =\lambda_{\overline{\alpha_{1}^{\prime} \cdot \beta_{1}^{\prime}}}\left(z^{\prime}\right) e^{i \psi \overline{\alpha_{1}^{\prime} \cdot \beta_{1}^{\prime}}}\left(z^{\prime}\right) \\
& =\left[1-\lambda_{\alpha_{1}^{\prime} \cdot \beta_{1}^{\prime}}\left(z^{\prime}\right)\right] e^{i\left[2 \pi-\psi_{\alpha_{1}^{\prime} \cdot \beta_{1}^{\prime}}\left(z^{\prime}\right)\right]} \\
& =\left[1-\lambda_{\alpha_{1}^{\prime}}\left(z^{\prime}\right) \cdot \lambda_{\beta_{1}^{\prime}}\left(z^{\prime}\right)\right] e^{i\left[2 \pi-2 \pi\left[\frac{\psi_{\alpha_{1}^{\prime}}\left(z^{\prime}\right)}{2 \pi} \cdot \frac{\psi_{\beta_{1}^{\prime}}\left(z^{\prime}\right)}{2 \pi}\right]\right]} \\
Y_{\overline{\alpha_{1}^{\prime} \cdot \beta_{1}^{\prime}}}\left(z^{\prime}\right) & =\left[1-\lambda_{\alpha_{1}^{\prime}}\left(z^{\prime}\right) \cdot \lambda_{\beta_{1}^{\prime}}\left(z^{\prime}\right)\right] e^{i\left[2 \pi-\frac{\psi_{\alpha_{1}^{\prime}}\left(z^{\prime}\right) \cdot \psi_{\beta_{1}^{\prime}}\left(z^{\prime}\right)}{2 \pi}\right]} .
\end{aligned}
$$

The R.H.S is given as:

$$
\begin{aligned}
Y_{\bar{\alpha}_{1}^{\prime}+\bar{\beta}_{1}^{\prime}}\left(z^{\prime}\right)= & \lambda_{\bar{\alpha}_{1}^{\prime}+\bar{\beta}_{1}^{\prime}}\left(z^{\prime}\right) e^{i \psi_{\bar{\alpha}_{1}^{\prime}+\bar{\beta}_{1}^{\prime}}\left(z^{\prime}\right)} \\
= & {\left[\lambda_{\bar{\alpha}_{1}^{\prime}}+\lambda_{\bar{\beta}_{1}^{\prime}}-\lambda_{\bar{\alpha}_{1}^{\prime}} \cdot \lambda_{\bar{\beta}_{1}^{\prime}}\right] e^{i 2 \pi\left[\frac{\psi_{\bar{\alpha}_{1}^{\prime}}\left(z^{\prime}\right)}{2 \pi}+\frac{\psi_{\bar{\beta}_{1}^{\prime}}\left(z^{\prime}\right)}{2 \pi}-\frac{\psi_{\bar{\alpha}_{1}^{\prime}}\left(z^{\prime}\right)}{2 \pi} \cdot \frac{\psi_{\bar{\beta}_{1}^{\prime}}\left(z^{\prime}\right)}{2 \pi}\right]} } \\
= & {\left[1-\lambda_{\alpha_{1}^{\prime}}\left(z^{\prime}\right)+1-\lambda_{\beta_{1}^{\prime}}\left(z^{\prime}\right)-\left[1-\lambda_{\alpha_{1}^{\prime}}\left(z^{\prime}\right)\right]\left[1-\lambda_{\beta_{1}^{\prime}}\left(z^{\prime}\right)\right]\right] } \\
& \left.e^{i 2 \pi\left[\frac{2 \pi-\psi}{\alpha_{1}^{\prime}}\left(^{\prime}\right)\right.}+\frac{2 \pi-\psi_{\beta_{1}^{\prime}}\left(z^{\prime}\right)}{2 \pi}-\frac{2 \pi-\psi_{\alpha_{1}^{\prime}}\left(z^{\prime}\right)}{2 \pi} \cdot \frac{2 \pi-\psi_{\beta_{1}^{\prime}}\left(z^{\prime}\right)}{2 \pi}\right] \\
Y_{\bar{\alpha}_{1}^{\prime}+\bar{\beta}_{1}^{\prime}}\left(z^{\prime}\right)= & {\left[1-\lambda_{\alpha_{1}^{\prime}}\left(z^{\prime}\right) \cdot \lambda_{\beta_{1}^{\prime}}\left(z^{\prime}\right)\right] e^{i\left[2 \pi-\frac{\psi_{\alpha_{1}^{\prime}}\left(z^{\prime}\right) \cdot \psi_{\beta_{1}^{\prime}}\left(z^{\prime}\right)}{2 \pi}\right]} . }
\end{aligned}
$$

From (1) and (2), we have

$$
Y_{\overline{\alpha_{1}^{\prime} \cdot \beta_{1}^{\prime}}}\left(z^{\prime}\right)=Y_{\bar{\alpha}_{1}^{\prime}+\bar{\beta}_{1}^{\prime}}\left(z^{\prime}\right)
$$

Thus

$$
\overline{\alpha_{1}^{\prime} \cdot \beta_{1}^{\prime}}=\bar{\alpha}_{1}^{\prime} \dot{+} \bar{\beta}_{1}^{\prime} .
$$


Proposition 4.9. If $\alpha_{1}^{\prime}$ and $\beta_{1}^{\prime}$ are two complex fuzzy sets on the universe of discourse $U$, then

$$
\left(\alpha_{1}^{\prime} \cdot \beta_{1}^{\prime}\right) \ominus \alpha_{1}^{\prime}=\phi .
$$

Proof. Let $Y_{\alpha_{1}^{\prime}}\left(z^{\prime}\right)=\lambda_{\alpha_{1}^{\prime}}\left(z^{\prime}\right) e^{i \psi_{\alpha_{1}^{\prime}}\left(z^{\prime}\right)}$ and $Y_{\beta_{1}^{\prime}}\left(z^{\prime}\right)=\lambda_{\beta_{1}^{\prime}}\left(z^{\prime}\right) e^{i \psi_{\beta_{1}^{\prime}}\left(z^{\prime}\right)}$ be the membership functions of $\alpha_{1}^{\prime}$ and $\beta_{1}^{\prime}$. Using the definition of dot product and bounded difference we will prove the following statement:

$$
Y_{\left(\alpha_{1}^{\prime} \cdot \beta_{1}^{\prime}\right) \ominus \alpha_{1}^{\prime}}\left(z^{\prime}\right)=\phi
$$

As

$$
\begin{aligned}
Y_{\left(\alpha_{1}^{\prime} \cdot \beta_{1}^{\prime}\right) \ominus \alpha_{1}^{\prime}}\left(z^{\prime}\right) & =\lambda_{\left(\alpha_{1}^{\prime} \cdot \beta_{1}^{\prime}\right) \ominus \alpha_{1}^{\prime}}\left(z^{\prime}\right) e^{i \psi}\left(\alpha_{1}^{\prime} \cdot \beta_{1}^{\prime}\right) \ominus \alpha_{1}^{\prime} \\
& =\max \left[0, \lambda_{\alpha_{1}^{\prime} \cdot \beta_{1}^{\prime}}\left(z^{\prime}\right)-\lambda_{\alpha_{1}^{\prime}}\left(z^{\prime}\right)\right] e^{i \max \left[0, \psi_{\alpha_{1}^{\prime} \cdot \beta_{1}^{\prime}}\left(z^{\prime}\right)-\psi_{\alpha_{1}^{\prime}}\left(z^{\prime}\right)\right]} \\
& =\max \left[0, \lambda_{\alpha_{1}^{\prime}}\left(z^{\prime}\right) \cdot \lambda_{\beta_{1}^{\prime}}\left(z^{\prime}\right)-\lambda_{\alpha_{1}^{\prime}}\left(z^{\prime}\right)\right] e^{i \max \left[0,2 \pi\left[\frac{\psi_{\alpha_{1}^{\prime}}\left(z^{\prime}\right)}{2 \pi} \cdot \frac{\psi_{\beta_{1}^{\prime}}\left(z^{\prime}\right)}{2 \pi}\right]-\psi_{\alpha_{1}^{\prime}}\left(z^{\prime}\right)\right]} \\
& =\max \left[0, \lambda_{\alpha_{1}^{\prime}}\left(z^{\prime}\right)\left[\lambda_{\beta_{1}^{\prime}}\left(z^{\prime}\right)-1\right]\right] e^{i \max \left[0, \psi_{\alpha_{1}^{\prime}}\left(z^{\prime}\right)\left[\frac{\psi_{\beta_{1}^{\prime}}\left(z^{\prime}\right)}{2 \pi}-1\right]\right]} \\
& =\max \left[0,-\lambda_{\alpha_{1}^{\prime}}\left(z^{\prime}\right) \lambda_{\bar{\beta}_{1}^{\prime}}\left(z^{\prime}\right)\right] e^{i \max \left[0,-\frac{\psi_{\alpha_{1}^{\prime}}\left(z^{\prime}\right) \psi_{\bar{\beta}_{1}^{\prime}}\left(z^{\prime}\right)}{2 \pi}\right]}, \\
Y_{\left(\alpha_{1}^{\prime} \cdot \beta_{1}^{\prime}\right) \ominus \alpha_{1}^{\prime}}\left(z^{\prime}\right) & =\phi .
\end{aligned}
$$

Thus we proved that

$$
\left(\alpha_{1}^{\prime} \cdot \beta_{1}^{\prime}\right) \ominus \alpha_{1}^{\prime}=\phi
$$

Proposition 4.10. If $\alpha_{1}^{\prime}$ and $\beta_{1}^{\prime}$ are two complex fuzzy sets on the universe of discourse $U$, then

$$
\left(\alpha_{1}^{\prime} \odot \bar{\beta}_{1}^{\prime}\right)=\left(\alpha_{1}^{\prime} \ominus \beta_{1}^{\prime}\right) .
$$

Proof. Let $Y_{\alpha_{1}^{\prime}}\left(z^{\prime}\right)=\lambda_{\alpha_{1}^{\prime}}\left(z^{\prime}\right) e^{i \psi_{\alpha_{1}^{\prime}}\left(z^{\prime}\right)}$ and $Y_{\beta_{1}^{\prime}}\left(z^{\prime}\right)=\lambda_{\beta_{1}^{\prime}}\left(z^{\prime}\right) e^{i \psi_{\beta_{1}^{\prime}}\left(z^{\prime}\right)}$ be the membership functions of $\alpha_{1}^{\prime}$ and $\beta_{1}^{\prime}$. Using the definition of bounded product, standard complex fuzzy complement and bounded difference we will prove the following statement:

$$
Y_{\alpha_{1}^{\prime} \odot \bar{\beta}_{1}^{\prime}}\left(z^{\prime}\right)=Y_{\alpha_{1}^{\prime} \ominus \beta_{1}^{\prime}}\left(z^{\prime}\right) \text {. }
$$

The L.H.S of the above statement is given as:

$$
\begin{aligned}
Y_{\alpha_{1}^{\prime} \odot \bar{\beta}_{1}^{\prime}}\left(z^{\prime}\right) & =\lambda_{\alpha_{1}^{\prime} \odot \bar{\beta}_{1}^{\prime}}\left(z^{\prime}\right) e^{i \psi_{\alpha_{1}^{\prime} \odot \bar{\beta}_{1}^{\prime}}\left(z^{\prime}\right)} \\
& =\max \left[0, \lambda_{\alpha_{1}^{\prime}}\left(z^{\prime}\right)+\lambda_{\bar{\beta}_{1}^{\prime}}\left(z^{\prime}\right)-1\right] e^{i \max \left[0, \psi_{\alpha_{1}^{\prime}}\left(z^{\prime}\right)+\psi_{\bar{\beta}_{1}^{\prime}}\left(z^{\prime}\right)-2 \pi\right]} \\
& =\max \left[0, \lambda_{\alpha_{1}^{\prime}}\left(z^{\prime}\right)+1-\lambda_{\beta_{1}^{\prime}}\left(z^{\prime}\right)-1\right] e^{i \max \left[0, \psi_{\alpha_{1}^{\prime}}\left(z^{\prime}\right)+2 \pi-\psi_{\beta_{1}^{\prime}}\left(z^{\prime}\right)-2 \pi\right]} \\
Y_{\alpha_{1}^{\prime} \odot \bar{\beta}_{1}^{\prime}}\left(z^{\prime}\right) & =\max \left[0, \lambda_{\alpha_{1}^{\prime}}\left(z^{\prime}\right)-\lambda_{\beta_{1}^{\prime}}\left(z^{\prime}\right)\right] e^{i \max \left[0, \psi_{\alpha_{1}^{\prime}}\left(z^{\prime}\right)-\psi_{\beta_{1}^{\prime}}\left(z^{\prime}\right)\right]} .
\end{aligned}
$$

The R.H.S of the above statement is given as:

$$
\begin{aligned}
Y_{\alpha_{1}^{\prime} \ominus \beta_{1}^{\prime}}\left(z^{\prime}\right) & =\lambda_{\alpha_{1}^{\prime} \ominus \beta_{1}^{\prime}}\left(z^{\prime}\right) e^{i \psi_{\alpha_{1}^{\prime} \ominus \beta_{1}^{\prime}}\left(z^{\prime}\right)} \\
Y_{\alpha_{1}^{\prime} \ominus \beta_{1}^{\prime}}\left(z^{\prime}\right) & =\max \left[0, \lambda_{\alpha_{1}^{\prime}}\left(z^{\prime}\right)-\lambda_{\beta_{1}^{\prime}}\left(z^{\prime}\right)\right] e^{i \max \left[0, \psi_{\alpha_{1}^{\prime}}\left(z^{\prime}\right)-\psi_{\beta_{1}^{\prime}}\left(z^{\prime}\right)\right]} .
\end{aligned}
$$


From (1) and (2),we have

$$
Y_{\alpha_{1}^{\prime} \odot \bar{\beta}_{1}^{\prime}}\left(z^{\prime}\right)=Y_{\alpha_{1}^{\prime} \ominus \beta_{1}^{\prime}}\left(z^{\prime}\right)
$$

Thus we proved that

$$
\left(\alpha_{1}^{\prime} \odot \bar{\beta}_{1}^{\prime}\right)=\left(\alpha_{1}^{\prime} \ominus \beta_{1}^{\prime}\right)
$$

Proposition 4.11. If $\alpha_{1}^{\prime}$ and $\beta_{1}^{\prime}$ are two complex fuzzy sets on the universe of discourse $U$, then

$$
\alpha_{1}^{\prime} \oplus \beta_{1}^{\prime}=\beta_{1}^{\prime} \oplus \alpha_{1}^{\prime} .
$$

Proposition 4.12. If $\alpha_{1}^{\prime}$ and $\beta_{1}^{\prime}$ are two complex fuzzy sets on the universe of discourse $U$, then

$$
\alpha_{1}^{\prime} \cup\left(\alpha_{1}^{\prime} \odot \beta_{1}^{\prime}\right)=\alpha_{1}^{\prime} \text {. }
$$

Proof. Let $Y_{\alpha_{1}^{\prime}}\left(z^{\prime}\right)=\lambda_{\alpha_{1}^{\prime}}\left(z^{\prime}\right) e^{\left.i \psi_{\alpha_{1}^{\prime}}{ }^{\prime}\right)}$ and $Y_{\beta_{1}^{\prime}}\left(z^{\prime}\right)=\lambda_{\beta_{1}^{\prime}}\left(z^{\prime}\right) e^{i \psi_{\beta_{1}^{\prime}}\left(z^{\prime}\right)}$ be the membership functions of $\alpha_{1}^{\prime}$ and $\beta_{1}^{\prime}$. Using the definition of complex fuzzy union and the bounded product we will prove the following statement:

$$
Y_{\alpha_{1}^{\prime} \cup\left(\alpha_{1}^{\prime} \odot \beta_{1}^{\prime}\right)}\left(z^{\prime}\right)=Y_{\alpha_{1}^{\prime}}\left(z^{\prime}\right) .
$$

The L.H.S of the above statement is given as

$$
\begin{aligned}
& Y_{\alpha_{1}^{\prime} \cup\left(\alpha_{1}^{\prime} \odot \beta_{1}^{\prime}\right)}\left(z^{\prime}\right)=\lambda_{\alpha_{1}^{\prime} \cup\left(\alpha_{1}^{\prime} \odot \beta_{1}^{\prime}\right)}\left(z^{\prime}\right) e^{i \psi_{\alpha_{1}^{\prime} \cup\left(\alpha_{1}^{\prime} \odot \beta_{1}^{\prime}\right)}\left(z^{\prime}\right)}=\max \left[\lambda_{\alpha_{1}^{\prime}}\left(z^{\prime}\right), \lambda_{\alpha_{1}^{\prime} \odot \beta_{1}^{\prime}}\left(z^{\prime}\right)\right] e^{i \max \left[\psi_{\alpha_{1}^{\prime}}\left(z^{\prime}\right), \psi_{\alpha_{1}^{\prime} \odot \beta_{1}^{\prime}}\left(z^{\prime}\right)\right]}, \\
& Y_{\alpha_{1}^{\prime} \cup\left(\alpha_{1}^{\prime} \odot \beta_{1}^{\prime}\right)}\left(z^{\prime}\right)=\max \left[\lambda_{\alpha_{1}^{\prime}}\left(z^{\prime}\right), \max \left[0, \lambda_{\alpha_{1}^{\prime}}\left(z^{\prime}\right)+\lambda_{\beta_{1}^{\prime}}\left(z^{\prime}\right)-1\right]\right] 1 e^{i \max \left[\psi_{\alpha_{1}^{\prime}}\left(z^{\prime}\right), \max \left[0, \psi_{\alpha_{1}^{\prime}}\left(z^{\prime}\right)+\psi_{\beta_{1}^{\prime}}\left(z^{\prime}\right)-2 \pi\right]\right]} .
\end{aligned}
$$

Also,

$$
Y_{\alpha_{1}^{\prime}}(z)=\lambda_{\alpha_{1}^{\prime}}\left(z^{\prime}\right) e^{i \psi_{\alpha_{1}^{\prime}}\left(z^{\prime}\right)}
$$

To prove the above statement, we have the following cases:

\section{Case I. If}

$$
\lambda_{\alpha_{1}^{\prime}}\left(z^{\prime}\right) \leq \lambda_{\beta_{1}^{\prime}}\left(z^{\prime}\right) \text { and } \psi_{\alpha_{1}^{\prime}}\left(z^{\prime}\right) \leq \psi_{\beta_{1}^{\prime}}\left(z^{\prime}\right),
$$

then from equation (1), we have

$$
\begin{aligned}
Y_{\alpha_{1}^{\prime} \cup\left(\alpha_{1}^{\prime} \odot \beta_{1}^{\prime}\right)}\left(z^{\prime}\right) & =\max \left[\lambda_{\alpha_{1}^{\prime}}\left(z^{\prime}\right), \lambda_{\alpha_{1}^{\prime}}\left(z^{\prime}\right)+\lambda_{\beta_{1}^{\prime}}\left(z^{\prime}\right)-1\right] e^{i \max \left[\psi_{\alpha_{1}^{\prime}}\left(z^{\prime}\right), \psi_{\alpha_{1}^{\prime}}\left(z^{\prime}\right)+\psi_{\beta_{1}^{\prime}}\left(z^{\prime}\right)-2 \pi\right]} \\
& =\lambda_{\alpha_{1}^{\prime}}\left(z^{\prime}\right) e^{i \psi_{\alpha_{1}^{\prime}}\left(z^{\prime}\right)} \\
Y_{\alpha_{1}^{\prime} \cup\left(\alpha_{1}^{\prime} \odot \beta_{1}^{\prime}\right)}\left(z^{\prime}\right) & =Y_{\alpha_{1}^{\prime}}\left(z^{\prime}\right) .
\end{aligned}
$$

Case II. If

$$
\lambda_{\alpha_{1}^{\prime}}\left(z^{\prime}\right) \geq \lambda_{\beta_{1}^{\prime}}\left(z^{\prime}\right) \text { and } \psi_{\alpha_{1}^{\prime}}\left(z^{\prime}\right) \geq \psi_{\beta_{1}^{\prime}}\left(z^{\prime}\right),
$$

then equation (1) implies that

$$
\begin{aligned}
Y_{\alpha_{1}^{\prime} \cup\left(\alpha_{1}^{\prime} \odot \beta_{1}^{\prime}\right)}\left(z^{\prime}\right) & =\max \left[\lambda_{\alpha_{1}^{\prime}}\left(z^{\prime}\right), \lambda_{\alpha_{1}^{\prime}}\left(z^{\prime}\right)+\lambda_{\beta_{1}^{\prime}}\left(z^{\prime}\right)-1\right] e^{i \max \left[\psi_{\alpha_{1}^{\prime}}\left(z^{\prime}\right), \psi_{\alpha_{1}^{\prime}}\left(z^{\prime}\right)+\psi_{\beta_{1}^{\prime}}\left(z^{\prime}\right)-2 \pi\right]}, \\
& =\lambda_{\alpha_{1}^{\prime}}\left(z^{\prime}\right) e^{i \psi_{\alpha_{1}^{\prime}}\left(z^{\prime}\right)} \\
Y_{\alpha_{1}^{\prime} \cup\left(\alpha_{1}^{\prime} \odot \beta_{1}^{\prime}\right)}\left(z^{\prime}\right) & =Y_{\alpha_{1}^{\prime}}\left(z^{\prime}\right) .
\end{aligned}
$$


Case III. If

$$
\lambda_{\alpha_{1}^{\prime}}\left(z^{\prime}\right) \leq \lambda_{\beta_{1}^{\prime}}\left(z^{\prime}\right) \text { and } \psi_{\alpha_{1}^{\prime}}\left(z^{\prime}\right) \geq \psi_{\beta_{1}^{\prime}}\left(z^{\prime}\right)
$$

then equation (1) implies that

$$
\begin{aligned}
Y_{\alpha_{1}^{\prime} \cup\left(\alpha_{1}^{\prime} \odot \beta_{1}^{\prime}\right)}\left(z^{\prime}\right) & =\max \left[\lambda_{\alpha_{1}^{\prime}}\left(z^{\prime}\right), \lambda_{\alpha_{1}^{\prime}}\left(z^{\prime}\right)+\lambda_{\beta_{1}^{\prime}}\left(z^{\prime}\right)-1\right] e^{i \max \left[\psi_{\alpha_{1}^{\prime}}\left(z^{\prime}\right), \psi_{\alpha_{1}^{\prime}}\left(z^{\prime}\right)+\psi_{\beta_{1}^{\prime}}\left(z^{\prime}\right)-2 \pi\right]} \\
& =\lambda_{\alpha_{1}^{\prime}}\left(z^{\prime}\right) e^{i \psi_{\alpha_{1}^{\prime}}\left(z^{\prime}\right)} \\
Y_{\alpha_{1}^{\prime} \cup\left(\alpha_{1}^{\prime} \odot \beta_{1}^{\prime}\right)}\left(z^{\prime}\right) & =Y_{\alpha_{1}^{\prime}}\left(z^{\prime}\right) .
\end{aligned}
$$

Case IV. If

$$
\lambda_{\alpha_{1}^{\prime}}\left(z^{\prime}\right) \geq \lambda_{\beta_{1}^{\prime}}\left(z^{\prime}\right) \text { and } \psi_{\alpha_{1}^{\prime}}\left(z^{\prime}\right) \leq \psi_{\beta_{1}^{\prime}}\left(z^{\prime}\right)
$$

then equation (1) implies that

$$
\begin{aligned}
Y_{\alpha_{1}^{\prime} \cup\left(\alpha_{1}^{\prime} \odot \beta_{1}^{\prime}\right)}\left(z^{\prime}\right) & =\max \left[\lambda_{\alpha_{1}^{\prime}}\left(z^{\prime}\right), \lambda_{\alpha_{1}^{\prime}}\left(z^{\prime}\right)+\lambda_{\beta_{1}^{\prime}}\left(z^{\prime}\right)-1\right] e^{i \max \left[\psi_{\alpha_{1}^{\prime}}\left(z^{\prime}\right), \psi_{\alpha_{1}^{\prime}}\left(z^{\prime}\right)+\psi_{\beta_{1}^{\prime}}\left(z^{\prime}\right)-2 \pi\right]} \\
& =\lambda_{\alpha_{1}^{\prime}}\left(z^{\prime}\right) e^{i \psi_{\alpha_{1}^{\prime}}\left(z^{\prime}\right)} \\
Y_{\alpha_{1}^{\prime} \cup\left(\alpha_{1}^{\prime} \odot \beta_{1}^{\prime}\right)}\left(z^{\prime}\right) & =Y_{\alpha_{1}^{\prime}}\left(z^{\prime}\right) .
\end{aligned}
$$

Thus from the above cases, we have

$$
\alpha_{1}^{\prime} \cup\left(\alpha_{1}^{\prime} \odot \beta_{1}^{\prime}\right)=\alpha_{1}^{\prime} .
$$

Example 4.13. Suppose

$$
\begin{aligned}
& \alpha_{1}^{\prime}=\frac{0.5 e^{i 1.2 \pi}}{-1}+\frac{0.4 e^{i 0.8 \pi}}{-2}+\frac{0.6 e^{i \pi}}{-3} \\
& \beta_{1}^{\prime}=\frac{0.7 e^{i 1.6 \pi}}{-1}+\frac{0.9 e^{i 1.3 \pi}}{-2}+\frac{0.8 e^{i 2 \pi}}{-3}
\end{aligned}
$$

are two complex fuzzy sets. Using CF union and bounded product we will prove the above property as:

$$
\begin{aligned}
\alpha_{1}^{\prime} \odot \beta_{1}^{\prime} & =\frac{0.2 e^{i 0.8 \pi}}{-1}+\frac{0.3 e^{i 0.1 \pi}}{-2}+\frac{0.4 e^{i \pi}}{-3} \\
\alpha_{1}^{\prime} \cup\left(\alpha_{1}^{\prime} \odot \beta_{1}^{\prime}\right) & =\left(\frac{0.5 e^{i 1.2 \pi}}{-1}+\frac{0.4 e^{i 0.8 \pi}}{-2}+\frac{0.6 e^{i \pi}}{-3}\right) \cup\left(\frac{0.2 e^{i 0.8 \pi}}{-1}+\frac{0.3 e^{i 0.1 \pi}}{-2}+\frac{0.4 e^{i \pi}}{-3}\right), \\
\alpha_{1}^{\prime} \cup\left(\alpha_{1}^{\prime} \odot \beta_{1}^{\prime}\right) & =\frac{0.5 e^{i 1.2 \pi}}{-1}+\frac{0.4 e^{i 0.8 \pi}}{-2}+\frac{0.6 e^{i \pi}}{-3} .
\end{aligned}
$$

Thus

$$
\alpha_{1}^{\prime} \cup\left(\alpha_{1}^{\prime} \odot \beta_{1}^{\prime}\right)=\alpha_{1}^{\prime} .
$$

Proposition 4.14. If $\alpha_{1}^{\prime}$ and $\beta_{1}^{\prime}$ are two complex fuzzy sets on the universe of discourse $U$, then

$$
\alpha_{1}^{\prime} \odot\left(\beta_{1}^{\prime} \cap \gamma_{1}^{\prime}\right)=\left(\alpha_{1}^{\prime} \odot \beta_{1}^{\prime}\right) \cap\left(\alpha_{1}^{\prime} \odot \gamma_{1}^{\prime}\right)
$$

Proof. The proof of this proposition is similar to the proof of Proposition 2. 
Example 4.15. Suppose

$$
\begin{aligned}
& \alpha_{1}^{\prime}=\frac{0.6 e^{i 1.2 \pi}}{-1}+\frac{0.9 e^{i 0.9 \pi}}{-2}+\frac{0.8 e^{i 1.7 \pi}}{-3} \\
& \beta_{1}^{\prime}=\frac{0.5 e^{i 1.6 \pi}}{-1}+\frac{0.3 e^{i 1.3 \pi}}{-2}+\frac{0.7 e^{i 1.3 \pi}}{-3} \\
& \gamma_{1}^{\prime}=\frac{0.9 e^{i \pi}}{-1}+\frac{0.5 e^{i 1.8 \pi}}{-2}+\frac{0.6 e^{i 0.6 \pi}}{-3}
\end{aligned}
$$

are two complex fuzzy sets. Using the bounded product and CF intersection we will prove the above property as:

$$
\begin{aligned}
\beta_{1}^{\prime} \cap \gamma_{1}^{\prime}= & \frac{0.5 e^{i \pi}}{-1}+\frac{0.3 e^{i 1.3 \pi}}{-2}+\frac{0.6 e^{i 0.6 \pi}}{-3} \\
\alpha_{1}^{\prime} \odot\left(\beta_{1}^{\prime} \cap \gamma_{1}^{\prime}\right)= & \left(\frac{0.6 e^{i 1.2 \pi}}{-1}+\frac{0.9 e^{i 0.9 \pi}}{-2}+\frac{0.8 e^{i 1.7 \pi}}{-3}\right) \odot\left(\frac{0.5 e^{i \pi}}{-1}+\frac{0.3 e^{i 1.3 \pi}}{-2}+\frac{0.6 e^{i 0.6 \pi}}{-3}\right), \\
& \alpha_{1}^{\prime} \odot\left(\beta_{1}^{\prime} \cap \gamma_{1}^{\prime}\right)=\frac{0.1 e^{i 0.2 \pi}}{-1}+\frac{0.2 e^{i 0.2 \pi}}{-2}+\frac{0.4 e^{i 0.3 \pi}}{-3} .
\end{aligned}
$$

Now

$$
\begin{gathered}
\alpha_{1}^{\prime} \odot \beta_{1}^{\prime}=\frac{0.1 e^{i 0.8 \pi}-\frac{0.2 e^{i 0.2 \pi}}{-2}+\frac{0.5 e^{i \pi}}{-3}}{\alpha_{1}^{\prime} \odot \gamma_{1}^{\prime}=\frac{0.5 e^{i 0.2 \pi}}{-1}+\frac{0.4 e^{i 0.7 \pi}}{-2}+\frac{0.4 e^{i 0.3 \pi}}{-3},} \\
\left(\alpha_{1}^{\prime} \odot \beta_{1}^{\prime}\right) \cap\left(\alpha_{1}^{\prime} \odot \gamma_{1}^{\prime}\right)=\frac{0.1 e^{i 0.2 \pi}}{-1}+\frac{0.2 e^{i 0.2 \pi}}{-2}+\frac{0.4 e^{i 0.3 \pi}}{-3} .
\end{gathered}
$$

From (1) and (2), we have

$$
\alpha_{1}^{\prime} \odot\left(\beta_{1}^{\prime} \cap \gamma_{1}^{\prime}\right)=\left(\alpha_{1}^{\prime} \odot \beta_{1}^{\prime}\right) \cap\left(\alpha_{1}^{\prime} \odot \gamma_{1}^{\prime}\right) .
$$

\section{Applications of complex fuzzy sets}

Here, we will discuss the applications of CFS in decision-making problems.

Definition 5.1. Let $U$ be a universal set then the complex fuzzy dominance matrix $S$ is a CFS on the product $E \times E$. It is defined by a membership function $Y_{S}$. The complex fuzzy dominance matrix $S$ is identified by a mapping $Y_{S}: E \times E \longrightarrow C F(U)$ where $C F(U)$ denotes the set of the complex fuzzy unit disk in $S$. Thus, $S$ can be denoted by the set of $n \times n$ matrix,

$$
S=\left(s_{i j}\right), s_{i j}=Y_{S}\left(e_{i}, e_{j}\right)=\lambda_{S}\left(e_{i}, e_{j}\right) e^{i \psi\left(e_{i}, e_{j}\right)}, \forall i, j \in[1,2, \ldots, k], i \neq j
$$

interpreted as the dominance degree of expert $e_{i}$ over $e_{j}$ on the set of

$$
\left(u_{i}, c_{j}\right), i \in\{1,2, \ldots, m\}, j \in\{1,2, \ldots, n\},
$$

where $s_{i j}=0$ denotes the indifference between $e_{i}$ and $e_{j}\left(e_{i} \sim e_{j}\right), s_{i j}>0$ denotes that $e_{i}$ is preferred to $e_{j}\left(e_{i} \succ e_{j}\right), s_{i j}<0$ denotes that $e_{j}$ is preferred to $e_{i}\left(e_{i} \prec e_{j}\right)$. Dominance degree of expert $e_{i}$ over $e_{j}$ on the set of $\left(u_{i}, c_{j}\right), i \in\{1,2, \ldots, m\}, j \in\{1,2, \ldots, n\}$ are calculated as,

$$
s_{i j}^{A, B}=\left(h_{i j}^{A}-h_{i j}^{B}\right), 1 \leq i \leq m, 1 \leq j \leq n,
$$


where $A$ and $B$ belong to the set of expert $E$ and $h_{i j}^{A}$ and $h_{i j}^{B}$ are complex fuzzy decision matrices of experts $A$ and $B$, respectively.

\section{Algorithm}

In this algorithm, we identify a maximum value by using multiple attribute decision-making problems. The following steps are used in this algorithm.

\section{Step I.}

Compute complex fuzzy decision matrices of a set of experts and for a multiple attribute decision making problem over $U$, a complex fuzzy decision matrix $H=\left(h_{i j}\right)$ can be written as:

$$
H=\left(h_{i j}\right)_{m \times n}=\left(\begin{array}{cccc}
h_{11} & h_{12} & \ldots & h_{1 n} \\
h_{21} & h_{22} & \ldots & h_{2 n} \\
\vdots & \vdots & & \vdots \\
h_{m 1} & h_{m 2} & \ldots & h_{m n}
\end{array}\right), h_{i j} \in[0,1] .
$$

\section{Step II.}

Complex fuzzy dominance matrices $S^{i}, i>0$ are built on the subtraction of complex fuzzy decision matrices $H^{j}, j=\{1,2, \ldots, k\}$ of individual experts.

$$
S^{i}=H^{j}-H^{l}, j=[1,2, \ldots, k], l=j+\epsilon, \epsilon>0 .
$$

\section{Step III.}

Compute the complex fuzzy dominance matrices $S^{i}, i>0$ are combined by the expression.

$$
S=\max _{l=1}^{q}\left(s_{i j}^{l}\right), i=[1,2, \ldots, m], j=[1,2, \ldots, n],
$$

where $q$ is the number of complex fuzzy dominance matrices.

\section{Step IV.}

Utilize equation (1), to calculate the correlation coefficients to multiple attribute decisionmaking problems.

$$
\left.q_{i}=\sum_{j=1}^{n} w_{j} \times s_{i j}, \quad i=1,2, \ldots, m\right) .
$$

\section{Step V.}

Rank the alternative according to these four attributes $q_{i}(i=1,2, \ldots, m)$. From the max values of $q_{i} ;(i=1,2, \ldots, m)$ identify $S$.

Example 5.2. Assume that a customer decides to purchase a new computer for his own private use. There are four alternatives $\left(M_{1}, M_{2}, M_{3}, M_{4}\right)$ with different production dates. The customer considers four attributes, namely $C_{1}$ (Performance), $C_{2}$ (Appearance), $C_{3}$ (Service), and $C_{4}$ (Experience) with the weight vector $w=(0.2,0.1,0.3,0.2)$ for selecting a computer. The customer wants to select a computer among the four alternatives above. Here, we use the principle of the minimum degree of difference between multiple attributes CFSs to solve the given steps.

\section{Step I.}

In the first step, construct the complex fuzzy decision matrices of three experts $A, B$, and $C$ and take the set of parameters $U=\left\{M_{1}, M_{2}, M_{3}, M_{4}\right\}$ and $C=\left\{C_{1}, C_{2}, C_{3}, C_{4}\right\}$ are

$$
A=\left(\begin{array}{llll}
0.8 & 0.7 & 0.5 & 0.9 \\
0.9 & 0.6 & 0.8 & 0.4 \\
0.6 & 0.9 & 0.5 & 0.7 \\
0.7 & 0.5 & 0.8 & 0.9
\end{array}\right), B=\left(\begin{array}{llll}
0.7 & 0.6 & 0.5 & 0.8 \\
0.6 & 0.4 & 0.6 & 0.3 \\
0.5 & 0.8 & 0.4 & 0.5 \\
0.4 & 0.3 & 0.7 & 0.8
\end{array}\right), C=\left(\begin{array}{cccc}
0.1 & 0.2 & 0.4 & 0.0 \\
0.2 & 0.1 & 0.3 & 0.0 \\
0.3 & 0.4 & 0.1 & 0.0 \\
0.2 & 0.3 & 0.3 & 0.0
\end{array}\right) .
$$




\section{Step II.}

Find the complex fuzzy dominance matrix $S^{i}, i>0$ are built of complex fuzzy decision matrices $H^{j}, j=\{1,2, \ldots, k\}$ and are calculated by,

$$
s_{i j}^{A, B}=\left(h_{i j}^{A}-h_{i j}^{B}\right), 1 \leq i \leq m, 1 \leq j \leq n,
$$

where $h_{i j}^{A}$ and $h_{i j}^{B}$ are complex fuzzy decision matrices of experts $A$ and $B$, respectively. The complex fuzzy dominance matrices between $A$ and $B$ is as follow:

$$
\begin{aligned}
s_{i j}^{A, B} & =\left(\begin{array}{llll}
0.1 & 0.1 & 0.0 & 0.1 \\
0.3 & 0.2 & 0.2 & 0.1 \\
0.1 & 0.1 & 0.1 & 0.2 \\
0.3 & 0.2 & 0.1 & 0.1
\end{array}\right), s_{i j}^{A, C}=\left(\begin{array}{llll}
0.7 & 0.5 & 0.1 & 0.9 \\
0.7 & 0.5 & 0.5 & 0.4 \\
0.3 & 0.5 & 0.4 & 0.7 \\
0.5 & 0.2 & 0.5 & 0.9
\end{array}\right), \\
s_{i j}^{B, C} & =\left(\begin{array}{llll}
0.6 & 0.4 & 0.1 & 0.8 \\
0.4 & 0.3 & 0.3 & 0.3 \\
0.2 & 0.4 & 0.3 & 0.5 \\
0.2 & 0.0 & 0.4 & 0.8
\end{array}\right) .
\end{aligned}
$$

\section{Step III.}

Find the complex fuzzy dominance matrix $S^{i}, i>0$ are combined by the expression

$$
S=\max _{l=1}^{q}\left(s_{i j}^{l}\right), i=[1,2, \ldots, m], j=[1,2, \ldots, n],
$$

where $q$ is the number of complex fuzzy dominance matrices.

$\begin{array}{lllll} & \mathrm{C}_{1} & \mathrm{C}_{2} & \mathrm{C}_{3} & \mathrm{C}_{4} \\ \mathrm{M}_{1} & 0.7 & 0.5 & 0.1 & 0.9 \\ \mathrm{M}_{2} & 0.7 & 0.5 & 0.5 & 0.4 \\ \mathrm{M}_{3} & 0.3 & 0.5 & 0.4 & 0.7 \\ \mathrm{M}_{4} & 0.5 & 0.2 & 0.5 & 0.9\end{array}$.

\section{Step IV.}

Utilize equation (1), to calculate the correlation coefficient to multiple attribute decisionmaking problem as follow:

$$
\begin{aligned}
& q_{1}=w_{1} s_{11}+w_{2} s_{21}+w_{3} s_{31}+w_{4} s_{41}=0.2 \times 0.7+0.1 \times 0.7+0.3 \times 0.3+0.2 \times 0.5 \\
& q_{1}=0.40
\end{aligned}
$$

Similarly,

$$
q_{2}=0.34, q_{3}=0.29, q_{4}=0.61
$$

Thus

$$
\left(\begin{array}{l}
q_{1} \\
q_{2} \\
q_{3} \\
q_{4}
\end{array}\right)=\left(\begin{array}{l}
0.40 \\
0.34 \\
0.29 \\
0.61
\end{array}\right)
$$

Step V.

Rank the alternative according to the relative attribute. Therefore $q_{4}>q_{1}>q_{2}>q_{3}$ indicates that the most desirable alternatives are $q_{4}$. 


\section{Conclusions}

In this paper, we discussed the basic set-theoretic operations and rules of set theory for generalized fuzzy sets with complexities. Also, we presented a few fundamental results on CFSs using bounded sum, bounded product, bounded difference, simple difference, Cartesian product, algebraic product, and algebraic sums. We also constructed a multiple attribute decision-making model, developed an algorithm with the help of a complex fuzzy dominance matrix, and used it in decision-making problems. We hope that our findings will help improve the research on fuzzy set theory and will open a new way for applications, especially in decision analysis.

\section{List of Abbreviations}

FS: Fuzzy Set; IFS: Intuitionistic Fuzzy Set; CFS: Complex Fuzzy Set.

\section{Declarations}

Availability of data and materials

Not applicable.

\section{Competing interests}

The authors declare that they have no competing interests.

\section{Funding}

This work is financially supported by the Higher Education Commission of Pakistan (Grant No: 7750/Federal/NRPU/R\&D/HEC/2017).

\section{Authorship Contributions}

All authors contributed equally.

\section{Acknowledgments}

Not applicable.

\section{References}

[1] M. Ali, F. Smarandache, Complex neutrosophic set, Neural Computing and Applications, 28 (2017), 1817-1834.

[2] K. Atanassov, Intuitionistic fuzzy sets, Fuzzy Sets and Systems, 20(1) (1986), 87-96.

[3] K. Atanassov, G. Gargov, Interval valued intuitionistic fuzzy sets, Fuzzy Sets and Systems, 31 (1989), 343-349.

[4] T. Aydın, S. Enginoğlu, Interval-valued intuitionistic fuzzy parameterized interval-valued intuitionistic fuzzy soft sets and their application in decision-making, Journal of Ambient Intelligence and Humanized Computing, 12(1) (2021), 1541-1558.

[5] E. Ayyildiz, A.T. Gumus, Interval-valued Pythagorean fuzzy AHP method-based supply chain performance evaluation by a new extension of SCOR model: SCOR 4.0, Complex and Intelligent Systems, 7(1) (2021), 559-576.

[6] F. Ecer, D. Pamucar, MARCOS technique under intuitionistic fuzzy environment for determining the COVID-19 pandemic performance of insurance companies in terms of healthcare services, Applied Soft Computing, 104 (2021), 107199. 
[7] P.A. Ejegwa, I.C. Onyeke, Intuitionistic fuzzy statistical correlation algorithm with applications to multicriteria-based decision-making processes, International Journal of Intelligent Systems, 36(3) (2021), 1386-1407.

[8] P. Ejegwa, S. Wen, Y. Feng, W. Zhang, N. Tang, Novel Pythagorean fuzzy correlation measures via Pythagorean fuzzy deviation, variance and covariance with applications to pattern recognition and career placement, IEEE Transactions on Fuzzy Systems, 2021.

[9] X. Gao, L. Pan, Y. Deng, Quantum Pythagorean fuzzy evidence theory (get): A negation of quantum mass function view, IEEE Transactions on Fuzzy Systems, 2021, Doi:10.1109/TFUZZ.2021.3057993.

[10] H. Garg, D. Rani, Novel similarity measure based on the transformed right-angled triangles between intuitionistic fuzzy sets and its applications, Cognitive Computation, 13(2) (2021), $447-465$.

[11] H. Garg, A novel accuracy function under interval-valued Pythagorean fuzzy environment for solving multicriteria decision making problem, Journal of Intelligent and Fuzzy Systems, 31(1) (2016), 529-540.

[12] W. Huang, F. Zhang, S. Xu, A complete ranking method for interval-valued intuitionistic fuzzy numbers and its applications to multicriteria decision making, Soft Computing, 25(3) (2021), 2513-2520.

[13] T. Mahmood, A novel approach towards bipolar soft sets and their applications, Journal of Mathematics, (2020), Doi: 10.1155/2020/4690808.

[14] S. Naz, M. Akram, Novel decision-making approach based on hesitant fuzzy sets and graph theory, Computational and Applied Mathematics, 38(1) (2019), 7.

[15] L. Pan, X. Gao, Y. Deng, K.H. Cheong, The constrained Pythagorean fuzzy sets and its similarity measure, IEEE Transactions on Fuzzy Systems, (2021), Doi: 0.1109/TFUZZ.2021.3052559.

[16] D. Ramot, R. Milo, M. Friedman, A. Kandel, Complex fuzzy sets, IEEE Transactions on Fuzzy Systems, 10(2) (2002), 171-186.

[17] D.E. Tamir, L. Jin, A. Kandel, A new interpretation of complex membership grade, International Journal of Intelligent Systems, 26 (2011), 285-312.

[18] V. Torra, Hesitant fuzzy sets, International Journal of Intelligent Systems, 25(6) (2010), 529539.

[19] Y. Xue, Y. Deng, H. Garg, Uncertain database retrieval with measure-based belief function attribute values under intuitionistic fuzzy set, Information Sciences, 546 (2021), 436-447.

[20] R.R. Yager, Pythagorean fuzzy subsets, In 2013 joint IFSA World Congress and NAFIPS Annual Meeting (IFSA/NAFIPS) (2013), 57-61.

[21] L. A. Zadeh, Fuzzy sets, Information and Control, 8 (1965), 338-353.

[22] L. A. Zadeh, The concept of a linguistic variable and its application to approximate reasoning, Information Sciences, 8(3) (1975), 199-249. 
[23] G. Zhang, S.T. Dillon, Y.K. Cai, J. Ma, J. Lu, Operation properties and $\delta$-equalities of complex fuzzy sets, International Journal of Approximate Reasoning, 50 (2009), 1227-1249.

[24] M. Zhao, G. Wei, C. Wei, J. Wu, TODIM method for interval-valued Pythagorean fuzzy MAGDM based on cumulative prospect theory and its application to green supplier selection, Arabian Journal for Science and Engineering, 46(2) (2021), 1899-1910. 